\title{
A MULTILEVEL APPROACH FOR COMPUTING THE LIMITED-MEMORY HESSIAN AND ITS INVERSE IN VARIATIONAL DATA ASSIMILATION
}

\author{
KIRSTY L. BROWN * ${ }^{*}$ IGOR GEJADZE ${ }^{\dagger}$, AND ALISON RAMAGE *
}

\begin{abstract}
Use of data assimilation techniques is becoming increasingly common across many application areas. The inverse Hessian (and its square root) plays an important role in several different aspects of these processes. In geophysical and engineering applications, the Hessian-vector product is typically defined by sequential solution of a tangent linear and adjoint problem; for the inverse Hessian, however, no such definition is possible. Frequently, the requirement to work in a matrix-free environment means that compact representation schemes are employed. In this paper, we propose an enhanced approach based on a new algorithm for constructing a multilevel eigenvalue decomposition of a given operator, which results in a much more efficient compact representation of the inverse Hessian (and its square root). After introducing these multilevel approximations, we investigate their accuracy and demonstrate their efficiency (in terms of reducing memory requirements and/or computational time) using the example of preconditioning a Gauss-Newton minimisation procedure.
\end{abstract}

Key words. data assimilation, inverse Hessian, limited memory, preconditioning, multigrid

AMS subject classifications. 65K05, 65K10, 15A09, 15A29

1. Introduction and background material. Methods of data assimilation (DA) have become an important tool for analysis of complex physical phenomena in various fields of science and technology. These methods allow us to combine mathematical models, data resulting from instrumental observations and prior information. In particular, variational approaches have proven to be particularly useful for solving high-dimensional DA problems arising in geophysical and engineering applications involving complex fluid flow models. The problems of variational DA can be formulated as optimal control problems (see, for example, $[12,28]$ ) to find unknown model parameters such as initial and/or boundary conditions, right-hand sides in the model equations (forcing terms), and distributed coefficients. Equivalently, variational DA can be considered as a special case of the maximum a posteriori probability (MAP) estimator in a Bayesian framework [10]. Variational DA, implemented in the form of incremental $4 D$-Var $[8,36]$, is currently a preferred method for operational forecasting in meteorology and oceanography (more recently also in the form of ensemble $4 D$-Var; see, for example, [29]).

The importance of the Hessian matrix and its inverse in variational DA for geophysical applications is underlined in [38], although this has been a well established fact for decades in areas of statistics such as non-linear regression (see, for example, [1]). Some relevant applications of the inverse Hessian are highlighted in $\S 1.2$. A special feature of working with the Hessian for very high-dimensional problems is that neither the Hessian nor its inverse can be directly accessed in matrix form. While the Hessian-vector product can be computed by solving a sequence of the tangent linear and adjoint problems, no such option exists for defining the inverse Hessian-vector product (or the inverse square root Hessian-vector product, which is also relevant in many applications). One obvious approach is therefore to consider limited-memory schemes for computing and storing the inverse Hessian (or its square root). In this

\footnotetext{
*Department of Mathematics and Statistics, University of Strathclyde, Glasgow G1 1XH, Scotland

${ }^{\dagger}$ IRSTEA-Montpellier, 361 Rue Jean Francois Breton, BP 5095, France. (igor.gejadze@irstea.fr). Questions, comments, or corrections to this document may be directed to that email address.
} 
context, the idea of a multilevel framework becomes relevant. This is due to the fact that the inverse Hessian is, in essence, an approximation of the posterior covariance matrix and, if the initial flow field is considered as a spatially distributed control, then the correlations of different lengths between the flow field values can be described at different levels of spatial discretisation.

Multigrid methods were initially developed for solving elliptic partial differential equations (PDEs) $[3,5]$ and have since been extended for solving PDEs of different types, see, for example, [41]. One key modern area of application of multigrid methods is in solving PDE-constrained optimisation or inverse problems, see the review paper [4]. Here the multigrid solver is applied directly to the optimality system, which includes the original model, its adjoint and the optimality condition. Some elements of the multigrid approach have been utilised previously in variational data assimilation algorithms in meteorology and oceanography, but a complete multigrid algorithm has been considered only recently in [11]. Multigrid methods can also used for solving eigenvalue problems, which is most relevant to the work presented here. The usual multigrid approach in this context is to treat the eigenvalue problem as a non-linear equation and apply a non-linear multigrid solver [7, 21, 24]. Alternatively, an outer eigenvalue solver such as Rayleigh quotient iteration can be employed, which requires the solution of systems of linear equations with a shifted coefficient matrix using multigrid as an inner solver [37]. A third approach uses a standard eigenvalue solver (such as Lanczos [26] or Arnoldi [2]) with multigrid as a preconditioner. This type of method is reviewed in [25].

In this paper we develop a general multilevel eigenvalue decomposition of a given symmetric operator. Given its spectral decomposition, an operator $A$, say, can be approximated by a finite number of its eigenvalues and eigenvectors. To achieve a desired approximation quality (in terms of a specified distance between the exact and approximated operators) a certain number of eigenpairs must be used, dependent on the eigenvalue distribution. However, for high-dimensional problems, the computationally feasible number of eigenpairs (in terms of available storage, for example) may be too small to achieve any useful approximation quality. Thus, a single level eigenvalue decomposition approach has its limitations. Our proposed multilevel eigenvalue decomposition algorithm (see §2) involves an outer multilevel loop that provides an incomplete eigenvalue decomposition (using Lanczos) of the operator at each level, resulting in a final approximation involving eigenpairs associated with each discretisation level. Note that, if $A$ is not symmetric, the same technique could be applied to $A^{T} A$.

The multilevel technique described in $\S 2$ allows us to build limited-memory approximations to $A^{-1}$ and $A^{-1 / 2}$ which, within a fixed memory framework, are much better than their single-level spectral counterparts. These approximations could be used in many situations, for example, as preconditioners for solving systems of linear equations, across multiple application areas: a specific example is given in $§ 2.3 .4$. In this paper, we use the technique as an efficient way of approximating the inverse Hessian in variational data assimilation. For operational DA problems, we also introduce a second idea, namely, decomposition of the Hessian into local sensor-based Hessians (see §3). Although this is distinct from the multilevel eigenvalue decomposition, the latter provides a framework for its practical implementation. In $\S 4$ we describe three different implementations of the algorithm, before exploring their accuracy and efficiency in $\S 5$. 
1.1. Data assimilation problem. We consider a non-linear evolution model with an unknown initial state (the analysis), which must be retrieved using some prior guess (the background) and incomplete observation data of the state evolution. Let $X$ be a Hilbert space of functions on a spatial domain $\Omega$ with associated inner product $\langle\cdot, \cdot\rangle_{X}$, and let $Y=L_{2}(0, T ; X)$ for some time interval $[0, T]$ with scalar product

$$
\langle f, g\rangle_{Y}=\int_{0}^{T}\langle f(t), g(t)\rangle_{X} d t, \quad \forall f, g \in Y .
$$

We will model a physical process on $\Omega$ that is described by a function $\varphi \in Y$ satisfying the evolution equation

$$
\left\{\begin{aligned}
\frac{\partial \varphi(t)}{\partial t} & =F(\varphi(t))+f(t), \quad t \in[0, T], \\
\varphi(0) & =u
\end{aligned}\right.
$$

where $u \in X, f \in Y$ and $F$ is a non-linear operator mapping $X$ into $X$. We assume that, for a given $u \in X$ and $f \in Y$ such that $\langle f, f\rangle_{Y}<\infty$, there exists a unique solution $\varphi \in Y$ to (1.1). For a particular $u$, the model (1.1) can also be represented in operator form via a control-to-state mapping $R$ such that

$$
\varphi=R(u)
$$

The above framework is very general: as a concrete example of a specific evolutionary process, in the numerical experiments in $\S 5$ we use Burgers' equation as a basic prototype equation of atmospheric dynamics.

Let $u_{\text {true }}$ be the true initial state in (1.1). We define the input data as follows. Firstly, we have a prior guess, or background function, $u_{b} \in X$ such that $u_{b}=u_{\text {true }}+$ $\xi_{b}$, where $\xi_{b} \in X$ is the background error. Secondly, we have some observations represented by a function $\varphi_{\text {obs }}$ in a finite-dimensional space $Y_{\text {obs }}=L_{2}\left(\mathbb{R}^{M}\right)$, called the observation space. Introducing the bounded linear observation operator $C: Y \rightarrow$ $Y_{o b s}$, we may write $\varphi_{o b s}=C\left(R\left(u_{\text {true }}\right)\right)+\xi_{o}$, where $\xi_{o} \in Y_{o b s}$ is the observation error. Assuming that $\xi_{b}$ and $\xi_{o}$ are normally distributed, unbiased and mutually uncorrelated, we define the covariance operators $V_{b}(\cdot)=E\left[\left\langle\cdot, \xi_{b}\right\rangle_{X} \xi_{b}\right]$ and $V_{o}(\cdot)=$ $E\left[\left\langle\cdot, \xi_{o}\right\rangle_{Y_{o b s}} \xi_{o}\right]$, where $E[\cdot]$ is the expectation. We further assume that the operators $V_{b}$ and $V_{o}$ are positive definite, hence invertible.

We now introduce the cost function

$$
J(u)=\frac{1}{2}\left\langle V_{b}^{-1}\left(u-u_{b}\right), u-u_{b}\right\rangle_{X}+\frac{1}{2}\left\langle V_{o}^{-1}\left(C R(u)-\varphi_{o b s}\right), C R(u)-\varphi_{o b s}\right\rangle_{Y_{o b s}},
$$

and formulate the following data assimilation (DA) problem with the aim of identifying the initial state in (1.1): for a given $f \in Y$, find $\bar{u} \in X$ such that the cost function (1.1) is minimised over all $u \in X$, that is,

$$
\bar{u}=\underset{u}{\arg \min } J(u) .
$$

We denote the error in the solution of this optimal control problem (the so-called analysis error) by $\delta u=\bar{u}-u_{\text {true }}$, and assume $\delta u$ is unbiased, that is, $E[\delta u]=0$, with the covariance operator $V_{\delta u}$ defined by $V_{\delta u}(\cdot)=E\left[\langle\cdot, \delta u\rangle_{X} \delta u\right]$. 
Assuming that the operator $F$ is continuously Fréchet differentiable, for functions $v \in X$ and $\psi \in Y$, the tangent linear model associated with (1.1) is given by

$$
\left\{\begin{aligned}
\frac{\partial \psi(t)}{\partial t} & =F^{\prime}(\varphi(t)) \psi(t) \\
\psi(0) & =v
\end{aligned}\right.
$$

(see, for example, [16]) where $\varphi(t) \equiv \varphi(t, u)$ depends on $u$. Recalling the control-tostate operator $R(u)$, and defining the associated tangent linear operator $R^{\prime}(u)$ for a given $u \in X$ by

$$
R^{\prime}(u) v=\lim _{\tau \rightarrow 0} \frac{R(u+\tau v)-R(u)}{\tau}, \forall v \in X,
$$

[30], we may write model (1.3) as $\psi=R^{\prime}(u) v$.

A key role in variational DA is assigned to the Hessian of the following auxiliary DA problem (see [16]): find $v \in X$ such that the cost function

$$
J_{1}(v)=\frac{1}{2}\left\langle V_{b}^{-1} v, v\right\rangle_{X}+\frac{1}{2}\left\langle V_{o}^{-1} C R(u) v, C R(u) v\right\rangle_{Y_{o b s}}
$$

is minimised over all $v \in X$. Defining the adjoint $R^{\prime *}(u)$ of the tangent linear operator $R^{\prime}(u)$ by

$$
\left\langle v, R^{\prime *}(u) v^{*}\right\rangle_{X}=\left\langle R^{\prime}(u) v, v^{*}\right\rangle_{Y}, \forall v \in X, \forall v^{*} \in Y,
$$

we may write the Hessian in operator form as

$$
\mathcal{H}(u)=V_{b}^{-1}+R^{\prime *}(u) C^{*} V_{o}^{-1} C R^{\prime}(u),
$$

where $C^{*}$ is adjoint to $C$. We denote the Hessian by $\mathcal{H}(u)$ to emphasise its dependence on $u$ through $\varphi$ in $R^{\prime}$ and $R^{\prime *}$. Note that, for functions $v \in X, \psi \in Y$ and $\psi^{*} \in Y$, the adjoint model $v=R^{\prime *}(u) \psi$ can be written in PDE form as

$$
\left\{\begin{aligned}
-\frac{\partial \psi^{*}(t)}{\partial t}-F^{\prime *}(\varphi(t)) \psi^{*}(t) & =\psi(t), \quad t \in(0, T) \\
\psi^{*}(T) & =0 \\
v & =\psi^{*}(0)
\end{aligned}\right.
$$

where $F^{\prime *}$ is adjoint to $F^{\prime}$.

1.2. The role of the Hessian and its inverse. The Hessian (1.7) and its inverse play important roles in different aspects of data assimilation. The first is as a coefficient matrix (and preconditioner) in incremental 4D-Var [8]. Here each step of an outer iterative Gauss-Newton process is of the form $u_{i+1}=u_{i}+\alpha_{i} \delta u_{i}$, with $u_{i}$ a discrete approximation of the unknown initial state at iteration $i$, descent step $\alpha_{i}$ and update (descent direction) $\delta u_{i}$. As the update satisfies

$$
\mathcal{H}\left(u_{i}\right) \delta u_{i}=-G\left(u_{i}\right),
$$

where $G\left(u_{i}\right)$ is the gradient of the cost function, a system of linear equations involving $\mathcal{H}$ has to be solved at each step. Given a Hessian-vector product evaluation routine, the systems in (1.9) are usually solved iteratively using, for example, the conjugate gradient (CG) algorithm [23]. An approximation of $\mathcal{H}^{-1}$, if available at a reasonable 
cost, can therefore be used to precondition equation (1.9) to accelerate convergence of this inner iteration. This is the application studied in our numerical experiments (see $§ 5.5)$.

In addition, $\mathcal{H}^{-1}$ is involved in several aspects of statistical post-processing and characterisation of the optimal solution. Firstly, for linear and moderately non-linear DA problems, $\mathcal{H}^{-1}$ can be used as an approximation of the analysis error covariance matrix $[16,35,38]$. For example, confidence intervals for the components of the analysis vector can be defined by the corresponding diagonal elements (variance) of $\mathcal{H}^{-1}(\bar{u})$. A column $c_{i}$ of $\mathcal{H}^{-1}(\bar{u})$ which includes the $i^{\text {th }}$ diagonal element can be obtained by solving the equation $\mathcal{H}(\bar{u}) c_{i}=e_{i}$ (where $e_{i}$ is a Euclidean unit vector). If the number of requested diagonal elements is significant, it would be much less expensive to evaluate $\mathcal{H}^{-1}(\bar{u})$ once and keep it in some limited-memory form, than to retrieve necessary diagonal elements using the Hessian-vector product rule. We note also that, as the Hessian $\mathcal{H}(\bar{u})$ is equivalent to the Fisher information matrix (up to a constant multiplier), the diagonal elements of the inverse Hessian can also be used in the context of optimal experimental design involving such optimality criteria as l-optimality, for example. Secondly, the analysis probability density function (pdf) is defined by the analysis $\bar{u}$ and the analysis error covariance. Random functions from the Gaussian distribution $\mathcal{N}\left(\bar{u}, \mathcal{H}^{-1}(\bar{u})\right)$ can therefore be used as 'particles' of the ensemble of initial states, which may be useful for ensemble forecasting [14, 40]. These functions can be generated using $u=\mathcal{H}^{-1 / 2}(\bar{u}) \xi$, where $\xi \sim \mathcal{N}(0, I)$, or using the eigenvalues of $\mathcal{H}(\bar{u})$ [13]. However, in highly non-linear cases the 'particles' generated using $\mathcal{H}$ are unlikely to belong to the true posterior distribution, thus one must solve perturbed DA problems. This approach is referred to as the fully non-linear ensemble method [16], or randomised maximum likelihood method [6]. In these cases, an approximation of $\mathcal{H}^{-1 / 2}$ can be used for preconditioning the non-linear minimisation process to accelerate convergence, often with impressive results. Lastly, the analysis error $\delta u$ and the data errors $\xi_{b}$ and $\xi_{o}$ are related via the approximate error equation

$$
\mathcal{H}(\bar{u}) \delta u=V_{b}^{-1} \xi_{b}+R^{\prime *}(\bar{u}) C^{*} \xi_{o}
$$

[18]. This equation can be considered as a meta-model for investigating the effects of non-Gaussian data errors on the analysis error pdf. Specifically, if the model depends on parameters $\theta \in \Theta$, where $\Theta$ is the parameter space, an important problem is to quantify the sensitivity of the analysis error to uncertainty $\xi_{\theta}$ in these parameters. This can be done using the relationship

$$
\mathcal{H}(\bar{u}) \delta u=R^{\prime *}(\bar{u}) C^{*} V_{o}^{-1} C D(\bar{u}) \xi_{\theta},
$$

where $D(\bar{u}): \Theta \rightarrow Y$. Once again, $\mathcal{H}$ must be inverted to obtain $\delta u$.

The applications listed above all involve either solving multiple systems of linear equations involving $\mathcal{H}$, or having access to the inverse operator $\mathcal{H}^{-1}$. In practice, an explicit discrete representation of $\mathcal{H}$ is never required, since the Hessian-vector product can be obtained by successively applying operators in formula (1.7. The development of feasible methods for generation, storage and subsequent use of $\mathcal{H}^{-1}$ or $\mathcal{H}^{-1 / 2}$ in this framework are not well understood: this is the prime motivation for our interest in the development of efficient algorithms for computing and managing the inverse Hessian such as those presented in this paper.

1.3. First-level preconditioning. In DA problems, it is common to transform the Hessian (1.7) to a new operator with a more favourable eigenvalue distribution 
using so-called first-level preconditioning with $V_{b}^{1 / 2}$. This results in the preconditioned Hessian

$$
H(u)=\left(V_{b}^{1 / 2}\right)^{*} \mathcal{H}(u) V_{b}^{1 / 2}=I+\left(V_{b}^{1 / 2}\right)^{*} R^{\prime *}(u) C^{*} V_{o}^{-1} C R^{\prime}(u) V_{b}^{1 / 2} .
$$

The action of applying $H(u)$ to a given function $v \in X$ is defined by the successive solutions of the following problems:

$$
\begin{gathered}
\left\{\begin{aligned}
\frac{\partial \psi(t)}{\partial t} & =F^{\prime}(\varphi(t, u)) \psi(t), \\
\psi(0) & =V_{b}^{1 / 2} v
\end{aligned}\right. \\
\left\{\begin{aligned}
-\frac{\partial \psi^{*}(t)}{\partial t}-F^{\prime *}(\varphi(u, t)) \psi^{*}(t) & =-C^{*} V_{o}^{-1} C \psi(t), \quad t \in(0, T) \\
\psi^{*}(T) & =0, \\
H(u) v & =v-V_{b}^{1 / 2} \psi^{*}(0) .
\end{aligned}\right.
\end{gathered}
$$

It can be seen from (1.10) that all eigenvalues of $H(\bar{u})$ are greater than or equal to one (a detailed analysis of the conditioning of $H(\bar{u})$ can be found in [20]). Furthermore, it has been observed that, for many practical DA problems, only a relatively small percentage of the eigenvalues are distinct enough from unity to contribute significantly to the Hessian. This suggests using limited-memory representations of the discrete Hessian, where this structure in the spectrum of $H$ is exploited. Specifically, a few leading eigenvalue/eigenvector pairs $\left\{\lambda_{i}, w_{i}\right\}$ are computed (typically using the Lanczos method $[9,26]$ as $H$ is available in vector-product form) and $H^{\alpha}$ is replaced by the approximation

$$
H^{\alpha}(u) \simeq I+\sum_{i=1}^{n}\left(\lambda_{i}^{\alpha}-1\right) w_{i} w_{i}^{*}
$$

where $\alpha$ is a real number (for example, $\alpha=-1$ or $\alpha=-1 / 2$ ), and the summation bound $n$ is much smaller than the dimension of the discrete Hessian $H$.

Although first-level preconditioning can be very helpful, it may not be sufficient in certain circumstances. Firstly, the number of eigenvalues of $H$ which are essentially distinct from unity depends on the observation impact, which is proportional to the number of observations and their accuracy. Taking into account the size of state and observation vectors used in modern realistic DA applications (usually $10^{9}-10^{12}$ in length for state and $10^{6}-10^{9}$ for observation vectors), the value of $m$ in (1.12) required to obtain a limited-memory approximation of reasonable quality may still be prohibitively large in terms of memory. Furthermore, in forecasting problems, any computational result has a lifespan, that is, a time period when this result remains usable. Given that each Lanczos iteration requires evaluating a Hessian-vector product (which involves running both the tangent linear and the adjoint models), the time needed for calculating even a small fraction of the spectrum could easily exceed the lifespan of the resulting approximation. We are therefore interested in gaining additional savings in memory and computing time over and above those afforded by approximation (1.12). For the remainder of the paper, we will consider only the projected Hessian $H(\bar{u})$ given by (1.10), that is, we assume that first-level preconditioning has already been applied. In what follows, we develop a new approximation to $H^{-1}$, based on a multilevel structure, which requires less memory or less computational time than (1.12). 
2. Multilevel eigenvalue decomposition algorithm. In this section we describe an algorithm for constructing a multilevel approximation to the inverse (and its square root) of a general symmetric positive definite operator $A$ associated with problem (1.1) (for example, $H(\bar{u})$ in $(1.10)$ ). Such approximations have many different practical uses, for example, as preconditioners for linear systems of equations, as representations of covariance matrices or in error/confidence interval analysis.

The key idea can be summarised as follows. Consider a limited-memory approximation to $A^{-1}$ of the form in (1.12) (with $\alpha=-1$ ). If we assume that $A$ is only available in operator-vector product form, that is, we can evaluate $A v$ for some discrete function $v$ on the underlying computational grid, the eigenvalues required can be calculated using the Lanczos method. Given a sequence of nested grids, a conceptual outline of the recursive multilevel process is as follows:

1. represent $A$ on the coarsest grid level;

2. use a local preconditioner to improve the eigenvalue distribution;

3. build a limited memory approximation to its inverse, which forms the basis of the local preconditioner at the next coarsest level;

4. move up one grid level and repeat.

As proof of concept, we describe the ideas in a one-dimensional setting, with commensurate numerical examples in $\S 5$. However, the concept is equally valid for twoand three-dimensional problems.

2.1. Multilevel grid structure. We consider the spatial domain $\Omega=[0,1]$ and construct a sequence of grids for discretising the DA problem described in $\S 1.1$. We suppose that the base grid on which the problem is defined has a uniform distribution of $m_{0}=m+1$ grid points, and use this as our finest grid (denoted by grid level $k=0$ ). We form the next grid by removing a grid point from in between each pair of existing points, to give a grid at level $k=1$ with $m_{1}=m / 2+1$ uniformly-spaced points. Continuing this refinement leads to a sequence of grids at levels $k=0,1,2, \ldots, k_{c}$ (where $k_{c}$ is the coarsest grid level), with the grid at level $k$ containing $m_{k}=m / 2^{k}+1$ grid points.

2.2. Grid transfer operators. We introduce the prolongation operator $S_{k, k-i}$, $0 \leq i \leq k$, which maps (interpolates) a discrete function $v_{k}$ defined at grid level $k$ to a finer grid level $k-i$ (or to the same grid for $i=0$ ). That is, the operator satisfies

$$
v_{k-i}=S_{k, k-i} v_{k}, \quad S_{k, k}=I_{k},
$$

where $I_{k}$ is the identity operator at grid level $k$. Similarly, the restriction operator $S_{k, k-i}^{*}$ maps a discrete function $v_{k-i}$ defined at grid level $k-i$ to a coarser grid level $k$. That is,

$$
\tilde{v}_{k}=S_{k, k-i}^{*} v_{k-i}, \quad S_{k, k}^{*}=I_{k},
$$

where $S_{k, k-i}^{*}$ is the adjoint operator to $S_{k, k-i}$. Combining operators (2.1) and (2.2) gives $\tilde{v}_{k}=S_{k, k-i}^{*} S_{k, k-i} v_{k}$, so $\tilde{v}_{k}=v_{k}$ only if

$$
S_{k, k-i}^{*} S_{k, k-i}=I_{k}
$$

In other words, for $\tilde{v}_{k}=v_{k}$ we require $S_{k, k-i}$ to be an orthonormal projection: we will refer to this situation as perfect interpolation.

To construct a multilevel representation of $A^{-1}$ and $A^{-1 / 2}$, we will also need the projection of the operator $A$ at a finer (or coarser) grid level. Let $A_{k}$ be a discrete 
representation of $A$ defined at grid level $k$ and suppose we want to find its projection at a finer grid level $k-i$ (we will denote this projection by $P_{k-i}\left(A_{k}\right)$ ). Given that a discrete function $v_{k}$ at level $k$ has been obtained by projecting the corresponding fine grid function $v_{k-i}, 0<i \leq k$ using the grid transfer operators described above, we consider what happens when $A_{k}$ is applied to this projection. Specifically, we decompose the fine grid function $v_{k-i}$ into two parts, and write $v_{k-i}=v_{k-i}^{(A)}+v_{k-i}^{(B)}$ with

$$
v_{k-i}^{(A)}=\left(I_{k-i}-S_{k, k-i} S_{k, k-i}^{*}\right) v_{k-i}, \quad v_{k-i}^{(B)}=S_{k, k-i} S_{k, k-i}^{*} v_{k-i} .
$$

Projecting each component separately to grid level $k$ gives a corresponding coarse grid function of the form $v_{k}=v_{k}^{(A)}+v_{k}^{(B)}$ where

$$
\begin{aligned}
v_{k}^{(A)}=S_{k, k-i}^{*} v_{k-i}^{(A)} & =S_{k, k-i}^{*}\left(I_{k-i}-S_{k, k-i} S_{k, k-i}^{*}\right) v_{k-i} \\
& =\left(S_{k, k-i}^{*}-\left(S_{k, k-i}^{*} S_{k, k-i}\right) S_{k, k-i}^{*}\right) v_{k-i},
\end{aligned}
$$

and

$$
v_{k}^{(B)}=S_{k, k-i}^{*} v_{k-i}^{(B)}=\left(S_{k, k-i}^{*} S_{k, k-i}\right) S_{k, k-i}^{*} v_{k-i} .
$$

If we now assume perfect interpolation, that is, $S_{k, k-i}$ satisfies (2.3), then (2.4) simplifies to $v_{k}^{(A)}=0$. In other words, $v_{k-i}^{(A)}$ contains modes of $v_{k-i}$ which are not supported on the coarser grid $k$ and, therefore, should not be transferred to this level at all. However, the second component $v_{k-i}^{(B)}$ must be projected to grid level $k$, the operator $A_{k}$ applied, and the result projected back to grid level $k-i$. Combining these two steps gives

$$
\begin{aligned}
P_{k-i}\left(A_{k}\right) v_{k-i} & =v_{k-i}^{(A)}+S_{k, k-i} A_{k} S_{k, k-i}^{*} v_{k-i}^{(B)} \\
& =\left(I_{k-i}-S_{k, k-i} S_{k, k-i}^{*}\right) v_{k-i}+S_{k, k-i} A_{k} S_{k, k-i}^{*} v_{k-i} \\
& =\left(S_{k, k-i}\left(A_{k}-I_{k}\right) S_{k, k-i}^{*}+I_{k-i}\right) v_{k-i}
\end{aligned}
$$

We therefore define the projection of $A_{k}$ at a finer grid level $k-i, 0 \leq i \leq k$ using

$$
\begin{aligned}
P_{k-i}\left(A_{k}\right) & =S_{k, k-i}\left(A_{k}-I_{k}\right) S_{k, k-i}^{*}+I_{k-i}, \quad 0<i \leq k, \\
P_{k}\left(A_{k}\right) & =A_{k} .
\end{aligned}
$$

Note that we will also need the adjoint of $P_{k-i}\left(A_{k}\right)$. It is easy to see $P_{k-i}^{*}\left(A_{k}\right)=$ $P_{k-i}\left(A_{k}^{*}\right)$.

In a similar way, we define the projection of an operator $A_{k-i}$ at a coarser grid level $k, 0 \leq i \leq k_{c}$ using

$$
\begin{aligned}
Q_{k}\left(A_{k-i}\right) & =S_{k, k-i}^{*}\left(A_{k-i}-I_{k-i}\right) S_{k, k-i}+I_{k}, \quad 0<i \leq k_{c} \\
Q_{k}\left(A_{k}\right) & =A_{k}
\end{aligned}
$$

with corresponding adjoint $Q_{k}^{*}\left(A_{k-i}\right)=Q_{k}\left(A_{k-i}^{*}\right)$.

Finally, we note that in the case of perfect interpolation the operator $P_{k-i}\left(A_{k}\right)$ in (2.5) has the important property that $P_{k-i}\left(A_{k}\right)=P_{k-i}\left(A_{k}^{1 / 2}\right) P_{k-i}\left(\left(A_{k}^{1 / 2}\right)^{*}\right)$. Also, the expression (2.6) simplifies to $Q_{k}\left(A_{k-i}\right)=S_{k, k-i}^{*} A_{k-i} S_{k, k-i}$. However, $Q_{k}\left(A_{k-i}\right) \neq$ $Q_{k}\left(A_{k-i}^{1 / 2}\right) Q_{k}\left(\left(A_{k-i}^{1 / 2}\right)^{*}\right)$. 
2.3. Multilevel algorithm. We now develop an algorithm for constructing a multilevel limited-memory representation of $A^{-1}$ (and $A^{-1 / 2}$ ) in operator-vector product form, that is, as $A^{-1} v$ or $A^{-1 / 2} v$. This is achieved by separating the eigensystem of operator $A$ into the subsystems associated with different representation levels. We assume that the operator-vector product is available at the finest grid level $k=0$, that is, we have available $A_{0} v_{0}$ for some fine grid function $v_{0}$. In $\S 2.3 .1$ we describe our algorithm based on a sequence of coarser grids $k=1,2, \ldots, k_{c}$, where $k_{c}$ is the coarsest level. One key ingredient of this algorithm is a sequence of local preconditioners applied at each grid level: these are described in detail in $§ 2.3 .2$.

2.3.1. Structure of the algorithm. We begin by representing the finest grid operator $A_{0}$ on level $k$ as $Q_{k}\left(A_{0}\right)$ using (2.6), then precondition this to obtain

$$
\tilde{Q}_{k}\left(A_{0}\right)=B_{k, k+1}^{*} Q_{k}\left(A_{0}\right) B_{k, k+1} .
$$

The level $k$ preconditioner $B_{k, k+1}$ will be chosen so that the eigenvalues of $\tilde{Q}_{k}\left(A_{0}\right)$ are closer to unity than those of $Q_{k}\left(A_{0}\right)$ : details of how this is done follow in $\S 2.3 .2$. We then use the Lanczos method to compute a specified number, $n_{k}$ say, of the largest eigenvalues of $\tilde{Q}_{k}\left(A_{0}\right)$ (measured in a log-squared sense, see $\S 5.4$ ), together with their associated eigenvectors. The resulting $n_{k}$ eigenpairs $\left\{\lambda_{\tilde{Q}}^{i}, U_{k}^{i}\right\}, i=1, \ldots, n_{k}$, are then used to construct a limited memory approximation to $\tilde{Q}_{k}\left(A_{0}\right)$, namely,

$$
\hat{Q}_{k}\left(A_{0}\right)=I_{k}+\sum_{i=1}^{n_{k}}\left(\lambda_{k}^{i}-1\right) U_{k}^{i}\left(U_{k}^{i}\right)^{T} .
$$

Note that, as in (1.12), an approximation to $\tilde{Q}_{k}\left(A_{0}\right)$ raised to any chosen power $\alpha$ is readily available. This means that $\hat{Q}_{k}^{-1}\left(A_{0}\right), \hat{Q}_{k}^{1 / 2}\left(A_{0}\right)$ and, most importantly for the preconditioners defined in the next section, $\hat{Q}_{k}^{-1 / 2}\left(A_{0}\right)$, are easily computed.

The accuracy of approximation (2.8) is clearly critically affected by the number of eigenvectors which are calculated and stored at each grid level. To facilitate later investigation of how these values should be chosen, we introduce the notation

$$
N_{e}=\left(n_{0}, n_{1}, \ldots, n_{k_{c}}\right), \quad \widehat{N_{e}}=\sum_{k=0}^{k_{c}} n_{k}
$$

for the vector containing these values for a particular approximation and the sum of its entries.

2.3.2. Level $k$ preconditioners. The algorithm above involves a preconditioner $B_{k, k+1}$ for $Q_{k}\left(A_{0}\right)$ local to the current grid level. The motivation for our choice of $B_{k, k+1}$ is the assumption that $Q_{k+1}\left(A_{0}\right)$ is a good approximation to $Q_{k}\left(A_{0}\right)$, so we can use the projection of the former to grid level $k$ to precondition the latter. That is, we expect that the eigenvalues of the preconditioned operator

$$
P_{k}\left(Q_{k+1}^{-1 / 2}\left(A_{0}\right)\right) Q_{k}\left(A_{0}\right) P_{k}\left(Q_{k+1}^{-1 / 2}\left(A_{0}\right)\right)
$$

to be clustered around 1. Furthermore, it can be seen from (2.7) and (2.8) that

$$
Q_{k}^{-1}\left(A_{0}\right)=B_{k, k+1} \tilde{Q}_{k}^{-1}\left(A_{0}\right) B_{k, k+1}^{*} \approx B_{k, k+1} \hat{Q}_{k}^{-1}\left(A_{0}\right) B_{k, k+1}^{*}
$$

and so

$$
Q_{k}^{-1 / 2}\left(A_{0}\right)=B_{k, k+1} \tilde{Q}_{k}^{-1 / 2}\left(A_{0}\right) \approx B_{k, k+1} \hat{Q}_{k}^{-1 / 2}\left(A_{0}\right) .
$$


Note that with this notation, preconditioner $B_{k, k+1}$ is applied on level $k$, using information projected from level $k+1$.

The above considerations are valid for grid levels $k=0, \ldots, k_{c}-1$. On the coarsest grid level $k=k_{c}$, grid level $k_{c}+1$ does not exist, so we set $B_{k_{c}, k_{c}+1}=I_{k_{c}}$. Note also that on the finest grid level $k=0$ we can use $A_{0}$ directly, that is, $Q_{0}\left(A_{0}\right) \equiv A_{0}$. In practice, moving from the coarsest to the finest grid, we accumulate the eigenpairs $\left\{\lambda_{k}, U_{k}\right\}, k=k_{c}, \ldots, 0$, in (2.8) which allows us to define the required products $A_{0}^{-1} v_{0}$ and $A_{0}^{-1 / 2} v_{0}$ via a recursive algorithm as follows. At a general grid level $k$, the preconditioner is constructed in a recursive way using information from the previous (coarser) grid levels via

$$
\begin{aligned}
& B_{k, k+1}= \begin{cases}P_{k}\left(B_{k+1, k+2} \hat{Q}_{k+1}^{-1 / 2}\left(A_{0}\right)\right) & k=0,1, \ldots, k_{c}-1 ; \\
I_{k_{c}}, & k=k_{c} ;\end{cases} \\
& B_{k, k+1}^{*}= \begin{cases}P_{k}\left(\hat{Q}_{k+1}^{-1 / 2}\left(A_{0}\right) B_{k+1, k+2}^{*}\right), & k=0,1, \ldots, k_{c}-1 ; \\
I_{k_{c}}, & k=k_{c} ;\end{cases}
\end{aligned}
$$

where

$$
\hat{Q}_{k}^{-1 / 2}\left(A_{0}\right)=I_{k}+\sum_{i=1}^{n_{k}}\left(\left(\lambda_{k}^{i}\right)^{-1 / 2}-1\right) U_{k}^{i}\left(U_{k}^{i}\right)^{*}
$$

(cf. $(2.8)$ ).

2.3.3. Summary. Using the above definitions, an operator representing the multilevel eigenvalue decomposition algorithm can be constructed as follows:

\section{Algorithm 2.1. Multilevel Eigenvalue Decomposition Algorithm}

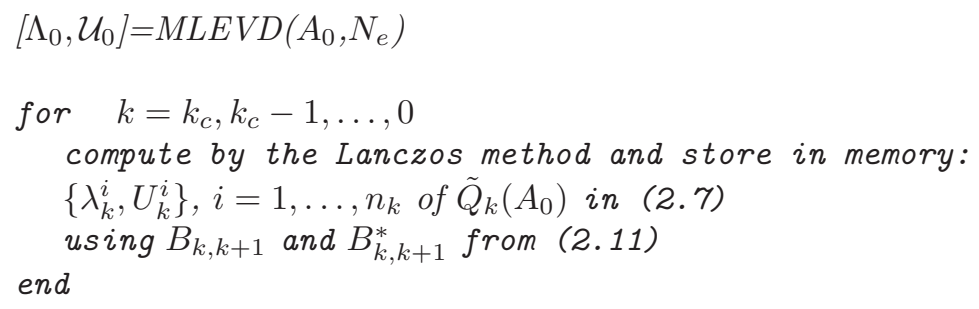

The input to this algorithm is $A_{0}$ (available in the form of an operator-vector product $A_{0} v_{0}$ at the finest level) together with the vector $N_{e}$ in (2.9) containing the number of eigenpairs to be calculated at each grid level. At a given level $k\left(\neq k_{c}\right)$, the algorithm uses the eigenpairs obtained at the previous (coarser) levels. The final output is a pair of vectors $\left[\Lambda_{0}, \mathcal{U}_{0}\right]$ containing the multilevel eigenstructure of $A_{0}$, which can be represented as follows:

$$
\begin{aligned}
\Lambda_{0} & =\left[\lambda_{k_{c}}^{1}, \ldots, \lambda_{k_{c}}^{n_{k_{c}}}, \lambda_{k_{c}-1}^{1}, \ldots, \lambda_{k_{c}-1}^{n_{k_{c}-1}}, \ldots, \lambda_{0}^{1}, \ldots, \lambda_{0}^{n_{0}}\right], \\
\mathcal{U}_{0} & =\left[U_{k_{c}}^{1}, \ldots, U_{k_{c}}^{n_{k_{c}}}, U_{k_{c}-1}^{1}, \ldots, U_{k_{c}-1}^{n_{k_{c}-1}}, \ldots, U_{0}^{1}, \ldots, U_{0}^{n_{0}}\right] .
\end{aligned}
$$

Given $\left[\Lambda_{0}, \mathcal{U}_{0}\right]$, for any function $v_{k}$ the products $Q_{k}^{-1}\left(A_{0}\right) v_{k}$ and $Q_{k}^{-1 / 2}\left(A_{0}\right) v_{k}$ can be recovered using (2.10), which in turn involves (2.11). In particular, for any fine grid function $v_{0}$ we can evaluate

$$
A_{0}^{-1} v_{0} \approx Q_{0}^{-1}\left(A_{0}\right)=B_{0,1} \hat{Q}_{0}^{-1}\left(A_{0}\right) B_{0,1}^{*} v_{0}
$$




$$
A_{0}^{-1 / 2} v_{0} \approx Q_{0}^{-1 / 2}\left(A_{0}\right)=B_{0,1} \hat{Q}_{0}^{-1 / 2}\left(A_{0}\right) v_{0},
$$

were $B_{0,1}$ and $B_{0,1}^{*}$ are defined in (2.11).

Using the notation in (2.9), we see that the vector $\Lambda_{0}$ in (2.12) contains a total of $\widehat{N_{e}}$ entries. As the grid at level $k$ contains $m_{k}=m / 2^{k}+1$ points, the vector $\mathcal{U}_{0}$ has a total of

$$
\sum_{k=0}^{k_{c}} n_{k} m_{k}=\left(\sum_{k=0}^{k_{c}} \frac{n_{k}}{2^{k}}\right) m+\widehat{N}_{e}
$$

entries. In what follows, we use the term "memory ratio" for the quantity

$$
r=\sum_{k=0}^{k_{c}} \frac{n_{k}}{2^{k}}
$$

as this gives a useful estimate of the ratio of the amount of storage required to $m$ (where the finest grid has $m+1$ points). An investigation of this storage requirement in practice for various eigenvalue combinations $N_{e}$ is given in $\S 5$.

We conclude this section by noting that Algorithm 2.1 can be generalised by using $\tilde{Q}_{k}\left(A_{k-i}\right)$ instead of $\tilde{Q}_{k}\left(A_{0}\right)$ in $(2.7)$, where $A_{k-i}, 1 \leq i \leq k$ is a direct representation of $A_{0}$ on grid level $k$. In particular, for the case of interest here (with $A_{0} \equiv H(u)$ ), we can define $H$ directly on a given level $k$ using

$$
H_{k}\left(u_{k}\right)=I_{k}+Q_{k}\left(\left(V_{b}^{1 / 2}\right)^{*}\right) R_{k}^{*}\left(u_{k}\right) Q_{k}\left(C^{*} V_{o}^{-1} C\right) R_{k}^{\prime}\left(u_{k}\right) Q_{k}\left(V_{b}^{1 / 2}\right),
$$

where $R_{k}\left(u_{k}\right)$ and $R_{k}^{*}\left(u_{k}\right)$ are the tangent linear (1.4) and adjoint (1.6) operators discretised on level $k$. Note that, as $V_{b}^{1 / 2}$ and $C^{*} V_{o}^{-1} C$ are defined at the finest grid level, appropriate projections are still required in (2.15). This approach allows the PDE problems defining the Hessian-vector product to be solved at any level $k-i$, while solving the eigenproblem at level $k$. This may help to reduce computational time, although the multilevel approximation will become less accurate (given the same allocated memory). We have not included an investigation of this approach in this paper.

2.3.4. Example of constructing a limited-memory inverse. For any symmetric positive definite operator $A$, the MLEVD algorithm in $\S 2.3 .3$ can be applied to obtain a multilevel limited-memory approximation of $A^{-1}$ or $A^{-1 / 2}$ (as in (2.13)). This approach can be particularly useful in the case where $A$ is a matrix representing a discretised differential operator, especially when the matrix $A$ is defined as a matrixvector product and solving multiple systems of equations involving $A$ is expected. In this section, we illustrate the usefulness of the technique by considering the particular example of approximating the inverse of a covariance matrix.

Consider a random vector $v$ of length $m$, resulting from the discretisation of a continuous function of one variable, and a multivariate Gaussian distribution $\mathcal{N}(\bar{v}, V)$. Here $\bar{v}$ is the mean vector and $V$ is the covariance matrix which, due to the high dimension of $v$, we assume is available in the matrix-vector product form $V v$. An example of such a product defined via the solution of the heat conduction equation 

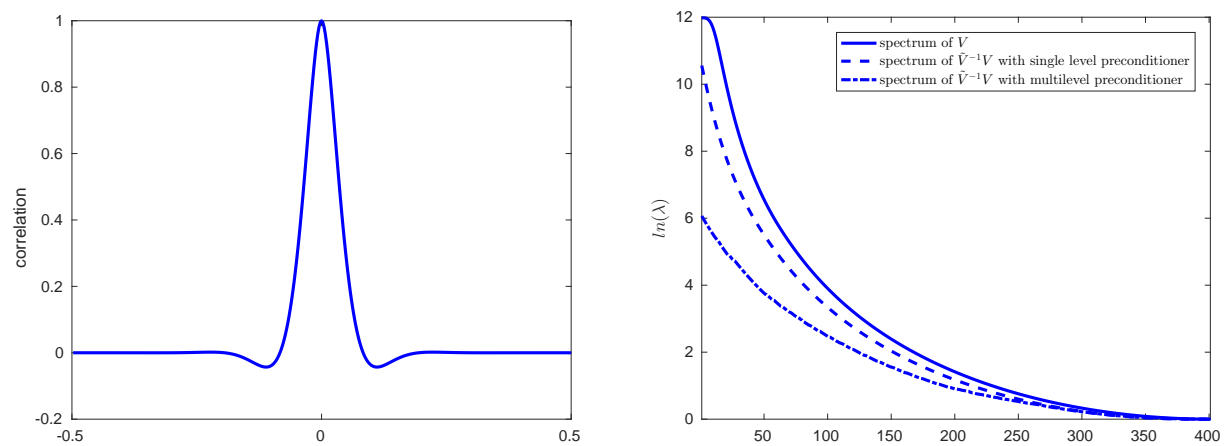

FIG. 2.1. The correlation function for $V$ (left) and the eigenvalues of $V$ (solid line), and $\tilde{V}^{-1} V$ with single-level (dotted line) and multi-level (dot-dash line) preconditioners (right).

can be seen in [31]. Checking the statistical hypothesis that a vector $v_{k}$ from an observed sample belongs to $\mathcal{N}(\bar{v}, V)$ relies on computing the Mahalanobis distance

$$
D_{M}=\left(v_{k}-\bar{v}\right)^{T} V^{-1}\left(v_{k}-\bar{v}\right) .
$$

As $V^{-1}$ is not readily available, obtaining $u=V^{-1}\left(v_{k}-\bar{v}\right)$ is usually done by solving the system of linear equations $V u=v_{k}-\bar{v}$ using an iterative method. This system itself needs a good preconditioner for $V$ in order to accelerate iterative convergence, that is, we require a matrix $\tilde{V}^{-1}$ such that the eigenvalue spectrum of $\tilde{V}^{-1} V$ facilitates faster convergence, either by clustering the eigenvalues, or reducing the condition number. In the numerical results below, we use both of these measures to evaluate preconditioner performance.

In this example, $V$ is a $401 \times 401$ covariance matrix with unit diagonal, characterised by the uniform correlation function $\tilde{V}(i, i-j)=\tilde{V}(i-j)$ (shown on the left of Figure 2.1). The eigenvalues of $V$ (plotted on a natural log scale) are represented by the solid line in the plot on the right of Figure 2.1. We compare them with the preconditioned spectrum of $\tilde{V}^{-1} V$ for two different preconditioners, both of which require the same amount of storage, as characterised by fixing the memory ratio $r$ in (2.14) (we use $r=16$ here). For the first preconditioner, all of the memory is deployed on the coarsest grid (with $m=401$ grid points): this corresponds to a single-level degenerate case of the MLEVD approximation with $N_{e}=(r, 0, \ldots, 0)$. The resulting preconditioned spectrum is represented by the dashed line in the right plot in Figure 2.1. In the second case, we use four discretisation levels for the MLEVD algorithm, with $N_{e}=(4,8,16,32)$, showing the resulting spectrum as the dash-dot line in the same plot. It is clear that this multilevel preconditioner outperforms the classical (one-level) spectral preconditioner here in terms of clustering the eigenvalues. Further results for different values of $r$ and $N_{e}$ are summarised in Table 2.1. As well as the condition number of $\tilde{V}^{-1} V$ (which should be compared to the unpreconditioned value of $1.6 e+5)$, the normalised Riemann distance $D$ between $V$ and $\tilde{V}$ is also tabulated (this is based on a comparison between the log-squared eigenvalues of the matrices, see §5.4). In each case, a slash is used to distinguish values obtained using the multilevel and single level approaches. Again, the multilevel MLEVD-based preconditioner is more effective. 


\begin{tabular}{cccc} 
Memory ratio $r$ & $N_{e}$ & Distance $D$ & Cond. number \\
\hline \hline 4 & $(1,2,4,8) /(4,0,0,0)$ & $0.84 / 0.96$ & $5.48 \mathrm{e}+4 / 1.59 \mathrm{e}+5$ \\
8 & $(2,4,8,16) /(8,0,0,0)$ & $0.71 / 0.92$ & $5.82 \mathrm{e}+3 / 1.41 \mathrm{e}+5$ \\
12 & $(3,6,12,24) /(12,0,0,0)$ & $0.61 / 0.86$ & $1.28 \mathrm{e}+3 / 7.68 \mathrm{e}+4$ \\
16 & $(4,8,16,32) /(16,0,0,0)$ & $0.54 / 0.81$ & $4.31 \mathrm{e}+2 / 3.84 \mathrm{e}+4$ \\
\hline \hline
\end{tabular}

TABLE 2.1

Comparison of multilevel and single level preconditioners for various values of memory ratio $r$ and eigenvalue combinations $N_{e}$.

3. Hessian decomposition. In the remainder of the paper, we focus on a specific application for the MLEVD algorithm, namely, the approximation of the inverse Hessian matrix (1.10) in variational data assimilation. Before we describe the specific algorithms proposed, we introduce a decomposition of the Hessian into a set of elementary Hessians which will prove to be useful later.

Using the factorisation

$$
V_{o}^{-1}=V_{o}^{-1 / 2} \bar{I} V_{o}^{-1 / 2},
$$

where $\bar{I}$ is the identity on the $M$-dimensional space $Y_{o b s}$ and $V_{o}^{-1 / 2}: Y_{o b s} \rightarrow Y_{o b s}$ is the spectral square-root of $V_{o}$, the preconditioned Hessian in (1.10) can be rewritten in the form

$$
H(u)=I+\left(V_{b}^{1 / 2}\right)^{*} R^{\prime *}(u) C^{*} V_{o}^{-1 / 2} \bar{I} V_{o}^{-1 / 2} C R^{\prime}(u) V_{b}^{1 / 2} .
$$

Now let $\mathcal{I}$ be a set of indices of the diagonal elements of $\bar{I}$, and suppose that $\mathcal{I}$ is partitioned into $L$ disjoint subsets $\mathcal{I}^{l}, l=1, \ldots, L$. Defining the diagonal matrices $\bar{I}^{l}$ such that

$$
\bar{I}_{i, i}^{l}=\left\{\begin{array}{ll}
1, & i \in \mathcal{I}^{l} \\
0, & i \notin \mathcal{I}^{l}
\end{array}, i=1, \ldots, M,\right.
$$

the identity can be written as

$$
\bar{I}=\sum_{l=1}^{L} \bar{I}^{l}
$$

Combining this with (3.1), after some algebraic manipulation we obtain a Hessian decomposition as follows:

$$
H(u)=I+\sum_{l=1}^{L}\left(H^{l}(u)-I\right)
$$

where

$$
H^{l}(u)=I+\left(V_{b}^{1 / 2}\right)^{*} R^{\prime *}(u) C^{*} V_{o}^{-1 / 2} \bar{I}^{l} V_{o}^{-1 / 2} C R^{\prime}(u) V_{b}^{1 / 2} .
$$

For a specific partition of $\mathcal{I}$, each elementary Hessian $H^{l}(u)$ can be presented in the limited-memory form (1.12) with the number of leading eigenpairs $n^{l}$ required for its accurate representation less than $n$, since

$$
\sum_{l=1}^{L} n^{l} \approx n .
$$


In particular, it is possible to define a partition of $\mathcal{I}^{l}$ such that $n^{l} \approx n / L$, with the number of Lanczos iterations and, correspondingly, the amount of CPU time needed for computing the eigenpairs of a single $H^{l}$ proportionally reduced. We also note that the elementary Hessians can be computed in parallel, so that the amount of CPU time required for computing the full limited-memory Hessian should not exceed the largest time spent on computing a single $H^{l}$.

To define an optimal partition, we may use the fact that the influence of the observations made by sensors located within a given spatial subdomain is also spatially localised. For example, suppose we have a set of $S$ sensors and let $\underline{x}(t)=$ $\left(x_{1}(t), x_{2}(t), \ldots, x_{S}(t)\right)^{T}$, where $x_{s}(t) \in \Omega$ represents the position of sensor $s$ at time $t$. Note that if $x_{s}(t)=x_{s}(0)$ for all $t \in[0, T]$, then sensor $s$ is stationary. Assuming that observations are recorded with a certain time period, we introduce an observation operator $C: Y \rightarrow Y_{o b s}$ such that

$$
C \varphi(t, x)=\varphi\left(t_{j}, x_{s}\left(t_{j}\right)\right), s=1, \ldots, S, j=1, \ldots, N,
$$

where $N$ is the number of observation nodes in time. The adjoint to $C, C^{*}: Y_{o b s} \rightarrow Y$, is given by

$$
C^{*} \varphi\left(t_{j}, x_{s}\left(t_{j}\right)\right)=\sum_{s=1}^{S} \sum_{j=1}^{N} \varphi\left(t_{j}, x_{s}\left(t_{j}\right)\right) \delta\left(x-x_{s}\left(t_{j}\right)\right) \delta\left(t-t_{j}\right),
$$

where $\delta$ is the Dirac delta function. We also assume that the observation covariance matrix $V_{o}$ is block-diagonal with $S$ diagonal blocks of dimension $N$. We now divide the spatial domain $\Omega$ into a set of disjoint subdomains $\Omega^{l}, l=1, \ldots L$ and define $\mathcal{I}^{l}$ to be the set of indices $i=(s-1) N+j$ such that $x_{s}\left(t_{j}\right) \in \Omega^{l}$, that is,

$$
\mathcal{I}^{l}=\left\{i \in \mathbb{N}, i=(s-1) N+j: x_{s}\left(t_{j}\right) \in \Omega^{l}\right\} .
$$

As long as the subdomains $\Omega^{l}$ are non-overlapping and cover the whole domain, decomposition (3.2)-(3.3) is defined for this partition. We will use this Hessian decomposition in $\S 4$ as part of a preconditioner for inner iterations of a Gauss-Newton method. In this context, we refer to an elementary Hessian $H^{l}$ as a local Hessian.

For a Hessian defined directly at a given level $k$ (as per (2.15)), it is easy to see that the local Hessian decomposition is

$$
H_{k}\left(u_{k}\right)=I_{k}+\sum_{l=1}^{L}\left(H_{k}^{l}\left(u_{k}\right)-I_{k}\right)
$$

where

$$
H_{k}^{l}\left(u_{k}\right)=I_{k}+Q_{k}\left(\left(V_{b}^{1 / 2}\right)^{*}\right) R_{k}^{\prime *}\left(u_{k}\right) Q_{k}\left(C^{*} V_{o}^{-1 / 2} \bar{I}^{l} V_{o}^{-1 / 2} C\right) R_{k}^{\prime}\left(u_{k}\right) Q_{k}\left(V_{b}^{1 / 2}\right) .
$$

For a specific partition of $\mathcal{I}$, powers of each elementary Hessian $H_{k}^{l}\left(u_{k}\right)$ can be approximated in limited-memory form as

$$
\left(\hat{H}_{k}^{l}\right)^{\alpha}\left(u_{k}\right) \simeq I_{k}+\sum_{i=1}^{n_{k}^{l}}\left(\left(\lambda_{k, i}^{l}\right)^{\alpha}-1\right) U_{k, i}^{l}\left(U_{k, i}^{l}\right)^{*}
$$

using the leading $n_{k}^{l}$ eigenpairs $\left\{\lambda_{k, i}^{l}, U_{k, i}^{l}\right\}, i=1, \ldots, n_{k}^{l}$ (cf. (1.12)) where the length of each eigenvector $U_{k, i}^{l}$ is equal to $m_{k}$. 
The calculation of a single local Hessian $H_{k}^{l}$ in (3.5) is a relatively inexpensive task which requires much less computational time than computing the global Hessian because each local Hessian deviates from the identity operator $I$ only within the area of influence surrounding the subdomain $\Omega^{l}$, with the size of this area depending on the transport mechanisms supported by the dynamical model. The number of eigenpairs used to describe this deviation could be relatively small, therefore a smaller number of Lanczos iterations may also be needed to evaluate the leading eigenvalues of $H_{k}^{l}$, with the corresponding eigenvectors being different from zero only within the local area of influence. Thus, a compact storage scheme for the eigenvectors can be utilised. To make further computational savings, the local Hessians $H_{k}^{l}$ can be computed at discretisation level $k=k^{\prime}>0$, as opposed to on the finest grid $k=0$. Finally, if the local Hessians are computed in parallel, then a very significant reduction in computing time can be achieved. Specifically, if the eigenvalue analysis for each $H_{k}^{l}$ can be carried out on an individual processor, the time needed for computing all $L$ eigenpair sets will be reduced to the maximum time taken to compute the eigenpairs of an individual local Hessian. We also observe that, for computing $H_{k}^{l}$, a local area model rather than the global model has to be run, and it is also possible that only some sensors from the whole observation array, or from only some areas of the computational domain, will be of interest so $H_{k}^{l}$ need not be calculated for every $\Omega^{l}$ : these ideas are not investigated further in this paper.

4. Approximating the inverse Hessian. In this section we use the multilevel eigenvalue decomposition in Algorithm 2.1 to build various approximations to the inverse Hessian $H^{-1}$, where $H$ is defined in (1.10). We describe three different possible algorithms, which may be useful depending on the constraints in place in terms of available computing time and memory. Some numerical experiments illustrating their relative accuracy and usefulness in practice are given in $\S 5$.

4.1. Algorithm 1. This involves using a straightforward application of the multilevel decomposition in Algorithm 2.1 to $H_{0}$, resulting in an eigenstructure $\left[\Lambda_{0}, \mathcal{U}_{0}\right]$ which can be used to evaluate $H_{0}^{-1} v_{0}$ and $H_{0}^{-1 / 2} v_{0}$ via (2.13). For a given optimal solution $\bar{u}_{0}$, the Hessian-vector product $H_{0} v_{0}$ is defined by (1.11) discretised at the finest grid level $k=0$. Appropriate values for the parameters in $N_{e}$ are discussed in $\S 5$. The algorithm can be outlined as follows:

Algorithm 4.1.

define $N_{e}$ compute $\left[\Lambda_{0}, U_{0}\right]=\operatorname{MLEVD}\left(H_{0}, N_{e}\right)$

4.2. Algorithm 2. In this approach, we assume that memory restrictions are not important, but that computing time is limited. To this end we utilise the Hessian decomposition idea as described in $\S 3$. This requires the choice of $L$ further parameters, $n_{k}^{l}, l=1, \ldots, L$, which determine the number of eigenpairs used in $\hat{H}_{k}^{l}$ (the limited memory approximation to $H_{k}^{l}$ in (3.5)). The algorithm can be outlined as follows: 
Algorithm 4.2.

define $N_{e}$, level $k^{\prime} \geq 0$, partition $\mathcal{I}^{l}, n_{k^{\prime}}^{l}, l=1, \ldots, L$

for $l=1, \ldots, L$ :

compute $\left\{\lambda_{k^{\prime}}^{i}, U_{k^{\prime}}^{i}\right\}^{l}, i=1, \ldots, n_{k^{\prime}}^{l}$ of $H_{k^{\prime}}^{l}$ in (3.5)

compute $\left[\Lambda_{0}, U_{0}\right]=\operatorname{MLEVD}\left(P_{0}\left(\hat{H}_{k^{\prime}}\right), N_{e}\right)$ where $\hat{H}_{k^{\prime}}$ is in form (3.4) based on

$\hat{H}_{k^{\prime}}^{l}$ from (3.6)

4.3. Algorithm 3. Although Algorithm 2 (in parallel form) requires less computational time than Algorithm 1, it requires more storage. In this third approach, we aim to alleviate this by reducing the memory requirements of Algorithm 2. We do that by applying MLEVD to the local inverse Hessians to obtain a reduced memory representation of the local Hessians before they are used in (3.4). The number of eigenpairs used in these additional approximations must now be specified: at level $k$, we denote these using a vector $N_{k}^{l}$ with an entry for the number of eigenvalues used on each (local) level, in an analogous way to (2.9) as

$$
N_{k}^{l}=\left(n_{k}^{l}, n_{k+1}^{l}, \ldots, n_{k_{c}}^{l}\right) .
$$

The algorithm can be outlined as follows:

Algorithm 4.3.

define $N_{e}$, level $k^{\prime}$, partition $\mathcal{I}^{l}, n_{k^{\prime}}^{l}, N_{k^{\prime}}^{l}, l=1, \ldots, L$

for $l=1, \ldots, L$ :

compute $\left\{\lambda_{k^{\prime}}^{i}, U_{k^{\prime}}^{i}\right\}^{l}, i=1, \ldots, n_{k^{\prime}}^{l}$ of $H_{k^{\prime}}^{l}$ in (3.5)

compute $\left[\Lambda_{k^{\prime}}, U_{k^{\prime}}\right]^{l}=\operatorname{MLEVD}\left(\left(\hat{H}_{k^{\prime}}^{l}\right)^{-1}, N_{k^{\prime}}^{l}\right)$, where $\left(\hat{H}_{k^{\prime}}^{l}\right)^{-1}$ is the limited-memory

inverse (1.12) of $H_{k^{\prime}}^{l}$ based on $\left\{\lambda_{k^{\prime}}^{i}, U_{k^{\prime}}^{i}\right\}^{l}$

compute $\left.\left[\Lambda_{0}, U_{0}\right]=\operatorname{MLEVD}\left(P_{0}\left(\hat{H}_{k^{\prime}}\right)\right), N_{e}\right)$ where $\hat{H}_{k^{\prime}}$ is in form (3.4) based on

multilvel approximations to $\hat{H}_{k^{\prime}}^{l}$ as defined by $\left\{\Lambda_{k^{\prime}}, U_{k^{\prime}}\right\}^{l}$ via (2.10a)

Note that the last step involves use of the recursive functions from (2.11), with recursion starting from level $k^{\prime}$.

5. Numerical Experiments. In this section we provide some implementation details and report the results of some numerical experiments which illustrate the performance of the three algorithms presented in the previous section. We begin by describing the model problems used in the rest of the section.

5.1. Model problems. As a test problem, we use as a model a one-dimensional Burgers' equation with a non-linear viscous term. Specifically, we consider

$$
\frac{\partial \varphi}{\partial t}+\frac{1}{2} \frac{\partial}{\partial x}\left(\varphi^{2}\right)=\frac{\partial}{\partial t}\left(\mu(\varphi) \frac{\partial \varphi}{\partial x}\right), \quad \varphi=\varphi(x, t), \quad t \in(0, T), \quad x \in(0,1)
$$

with the Neumann boundary conditions $\frac{\partial}{\partial x}(\varphi(0, t))=\frac{\partial}{\partial x}(\varphi(1, t))=0$ and viscosity coefficient

$$
\mu(\varphi)=\mu_{0}+\mu_{1}\left(\frac{\partial \varphi}{\partial x}\right)^{2}
$$

where $\mu_{0}$ and $\mu_{1}$ are positive constants (in all computations we use $\mu_{0}=10^{-4}$ and $\mu_{1}=10^{-5}$ ). The corresponding analytic expressions for the tangent linear and adjoint 
models are presented in Appendix A. Burgers' equation is often considered for testing data assimilation algorithms as a simple model describing elements of atmospheric flow motion [15].

We use an implicit time discretisation and the power law first-order finite volume scheme for spatial discretisation [34]. At each time step, we perform non-linear iterations to converge on non-linear coefficients. The tangent linear (1.3) and adjoint (1.8) models are obtained by using the automatic differentiation engine Tapenade [22], applied to the FORTRAN code implementing the above presented model.

We use two different initial states, defined by

$$
\begin{gathered}
u_{\text {true }}^{1}(x)=0.1+0.35\left[1+\sin \left(4 \pi x+\frac{3 \pi}{2}\right)\right], \quad 0<x<1 \\
u_{\text {true }}^{2}(x)= \begin{cases}0.5[1-\cos (8 \pi x)], & 0<x \leq 0.4, \\
0.5[\cos (4 \pi(x-1))-1], & 0.6 \leq x<1, \\
0 & \text { otherwise }\end{cases}
\end{gathered}
$$

The corresponding flow evolutions are presented in Figure 5.1.
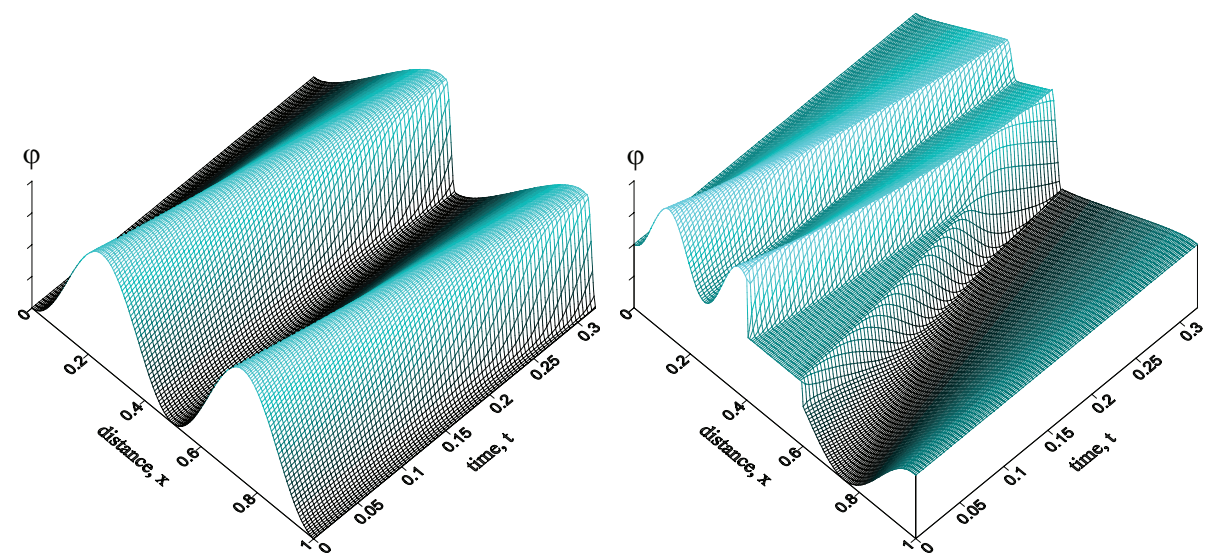

FIG. 5.1. Flow evolution for initial states (5.2): $u_{\text {true }}^{1}$ (left) and $u_{\text {true }}^{2}$ (right).

We also consider two different configurations of the sensors which provide the observations to be assimilated. For this one-dimensional problem, Scheme A has seven stationary sensors, fixed at points $0.3,0.4,0.45,0.5,0.55,0.6$, and 0.7 in $[0,1]$. In Scheme B, there is one moving sensor which traverses the domain $[0,1]$ from left to right twice during the observation time (see below for implementation details). This is to emulate satellite observations. The combinations used in our four model problems are detailed in Table 5.1.

5.2. Miscellaneous implementation details. The multilevel structure comprises four grids, with 401 grid points on the finest grid level $(k=0)$ and 51 points on the coarsest grid level $(k=3)$. We use cubic splines to implement the prolongation operator $S_{k, k-i}$ in $(2.1)$, with the subroutine for its adjoint operator $S_{k, k-i}^{*}$ (restriction) again obtained by using automatic differentiation. For test cases MP1 and MP2 (stationary sensors positioned as listed above), each local Hessian $H^{l}$ corresponds to one sensor located at $x=x^{l}$. For test cases MP3 and MP4 (moving 


\begin{tabular}{ccc} 
Model problem & Observation scheme & Initial condition \\
\hline \hline MP1 & $\mathrm{A}$ & $u_{\text {true }}^{1}$ \\
MP2 & $\mathrm{A}$ & $u_{\text {true }}^{2}$ \\
MP3 & $\mathrm{B}$ & $u_{\text {true }}^{1}$ \\
MP4 & $\mathrm{B}$ & $u_{\text {true }}^{2}$ \\
\hline \hline
\end{tabular}

TABLE 5.1

Details of the four model problems studied.

sensor), the spatial domain $\Omega=(0,1)$ is divided into six subdomains confined by points $(0,0.2,0.4,0.5,0.6,0.8,1.0)$ and each local Hessian takes into account only measurements inside subdomain $\Omega_{l}$.

The background error covariance matrix $V_{b}$ is defined under the assumption that the background error belongs to the Sobolev space $W_{2}^{2}[0,1]$ (see [17], Section 5.1). For each sensor configuration, the background error standard deviation can be seen later in Figure 5.3, where the correlation radius is uniform in space and equal to 0.2. The observation error is Gaussian, uncorrelated, and has standard deviation $\sigma_{o}=0.016$. Thus, $V_{o}$ is diagonal with uniform variance $\left(V_{o}\right)_{i, i}=\sigma_{o}^{2}$.

The eigenvalue problems are solved using standard ARPACK [27] to implement the implicitly restarted Lanczos method. We note, that although calculating the eigenvalues of $\tilde{Q}_{k}\left(A_{0}\right)$ by the Lanczos method has to be performed in double precision, single precision storage of the results would be sufficient in practice because all eigenpairs calculated at level $k$ are involved in preconditioning of $\tilde{Q}_{k-1}\left(A_{0}\right)$, so round-off errors introduced at level $k$ are largely compensated for at level $k-1$.

5.3. Preliminary results. In what follows, we interpret the inverse Hessian as a posterior covariance matrix. By the standard deviation (sd), here we mean a vector of the square roots of the diagonal elements of the inverse Hessian (variance). The correlation matrix $\bar{V}$ is the inverse Hessian matrix symmetrically scaled to have unit diagonal. That is, it has entries

$$
\bar{V}_{i j}=\frac{\mathcal{H}_{i j}^{-1}}{\mathcal{H}_{i i}^{-1 / 2} \mathcal{H}_{j j}^{-1 / 2}} .
$$

Figure 5.2 shows a plot of the correlation matrix for MP1 before (left) and after (right) first-level preconditioning is applied. Note that the (unit) diagonal entries have been omitted here to show the detail of the matrix structure. Although first-level preconditioning reduces a lot of the off-diagonal entries, what remains still deviates significantly from the identity, so there is scope for further preconditioning.

Figure 5.3 shows the standard deviation (as defined above), plotted as a function of $x$ on $[0,1]$. The top plots correspond to MP1 (stationary sensors), and the bottom plots to MP3 (moving sensor). In the left plots, the background sd is shown (labelled bg), along with the analysis sd based on $\mathcal{H}_{k}, k=0, \ldots, 4$. The sd which corresponds to $k=0\left(\mathcal{H}_{0}\right.$ is the Hessian (1.7) defined on the finest discretisation level) can be used as a reference function value for comparing with sd estimates based on $\mathcal{H}_{k}, k>0$ (defined in an analogous way to $H_{k}$ in (2.15)). To make a meaningful comparison, we consider $\mathcal{H}_{k}$ projected to the finest representation level, that is, $P_{0}\left(\mathcal{H}_{k}\right)$. The right plots in Figure 5.3 correspond to the equivalent sd values where first-level preconditioning has been applied. 

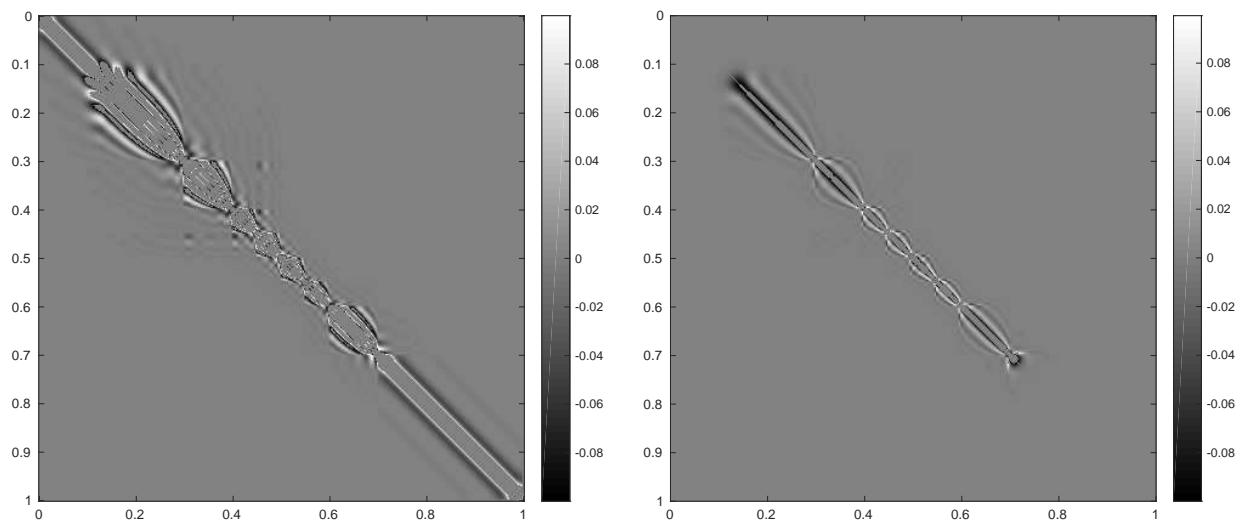

FIG. 5.2. Correlation matrix for MP1 before (left) and after (right) first-level preconditioning has been applied. Unit diagonal entries have been omitted.

These results substantiate the key idea of our approach, namely, that the inverse Hessian at level $k-1$ projected to level $k$ could be a good preconditioner for the Hessian at level $k$. Also, comparing the top and bottom subplots, an important difference between the impact made by stationary and moving sensors is apparent. A stationary sensor injects all information at one point, then the information is propagated over the spatial domain solely by the adjoint model. As a result, the variance at sensor location point tends towards the observation error variance $\sigma_{o}^{2}$. In contrast, a moving sensor injects a single piece of information at the current location point, which does not result in a sharp reduction of the local variance, then it moves to another point, thus spreading information throughout the domain. In this case the distribution of information is less dependent on the model transport, which itself depends on the discretisation of operators $R^{\prime}(\bar{u})$ and $R^{\prime *}(\bar{u})$. Thus, the coarse grid approximations to the analysis variance should be better than in case of stationary sensors, as can be observed in Figure 5.3. Furthermore, the difference in the moving sensor case has a low-frequency nature, which can be accommodated by a relatively low number of eigenpairs. This explains why our approach is more efficient for an observation scheme involving moving sensors, which will be apparent in the numerical results presented in $\S 5.5$.

5.4. Investigating approximation accuracy. In the first set of experiments, we apply Algorithm 1 described in $\S 4.1$ to $H\left(\equiv H_{0}\right)$ and use the resulting multilevel eigenvalue decomposition (2.12) to build a low-memory approximation to $H^{-1}$ in recursive form (2.13), which we will denote by $\tilde{H}^{-1}$. To assess the accuracy of our approximations, we measure the difference between two matrices in terms of the Riemann distance. That is, for two symmetric positive definite $n \times n$ matrices $A$ and $B$, we define

$$
\delta(A, B)=\left\|\ln \left(B^{-1} A\right)\right\|_{F}=\left(\sum_{i=1}^{n} \ln ^{2} \lambda_{i}\right)^{1 / 2}
$$



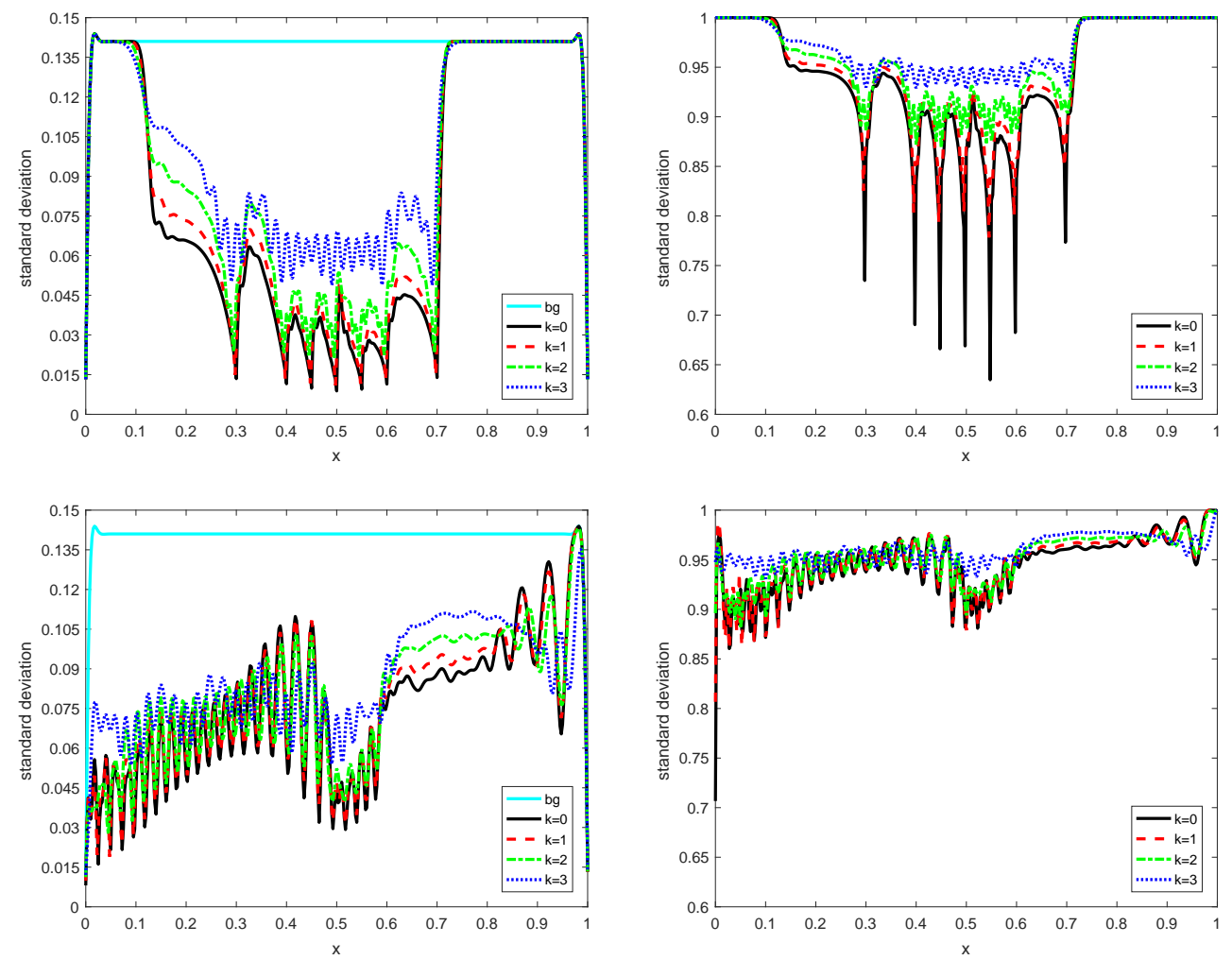

FIG. 5.3. Standard deviation before (left) and after (right) first-level preconditioning is applied. The top plots correspond to MP1 (stationary sensors), and the bottom plots to MP3 (moving sensor).

where $\lambda_{i}, i=1, \ldots, n$ are the eigenvalues of $B^{-1} A$ (see, for example, [32]). The Riemann distance can be considered as a symmetric measure of the difference between two Gaussian probability distributions having equal modes. Since the inverse Hessian is an approximation of the analysis error covariance matrix, it is very natural to use this measure in the current context. We will compare matrices after we have applied the first-level preconditioning described in $\S 1.3$ : it is easily shown that the Riemann distance remains unchanged on applying symmetric preconditioning to $A$ and $B$, so the distance between two symmetric positive definite matrices in the original and projected spaces is the same.

The accuracy of a given approximation will clearly be critically dependent on the number of eigenvalues calculated at each grid level (that is, the choice of $N_{e}=$ $\left.\left(n_{0}, n_{1}, n_{2}, n_{3}\right)\right)$. Ideally, we would like to be able to identify the 'optimal' combination $N_{e}$ for a given problem but this is non-trivial, particularly as the definition of optimality is itself dependent on the problem constraints. For example, one may want the best approximation which can be stored given a set amount of memory, or perhaps the approximation which ensures the biggest reduction in CPU time for computing $\tilde{H}^{-1} v$. Here we will focus on the former approach, and assume that there is a fixed memory ratio $r$ (see (2.14)) allowed for a particular problem. Within this 
fixed-memory framework, we measure the normalised Riemann distance

$$
D=\frac{\delta\left(H^{-1}, \tilde{H}^{-1}\right)}{\delta\left(H^{-1}, I\right)}
$$

where $I$ is an appropriately-sized identity matrix. By evaluating $D$ for approximations constructed using all possible eigenvalue combinations $N_{e}$ for a given memory ratio $r$, we can identify the best combinations $N_{e}$ for this fixed memory problem.

Let us consider case MP1. If $\tilde{H}^{-1}$ is constructed from 64 eigenpairs of $H^{-1}$ (corresponding to memory ratio $r=64$ ), a good approximation with $D=2.98 e-4$ is achieved. However, if available memory is restricted to $r=8$, so only 8 eigenpairs at level 0 can be stored, the resulting approximation is very poor $(D=7.71 e-1)$. However, if $\tilde{H}^{-1}$ is constructed using the multilevel eigenvalue decomposition, much better approximations can be achieved. The eigenvalues of $H^{-1}$ and $\tilde{H}^{-1}$ for MP1 with two representative eigenvalue combinations for $r=8$ are illustrated in Figure 5.4. In each subplot, the first 70 eigenvalues of $H^{-1}$ are plotted as blue circles (the rest
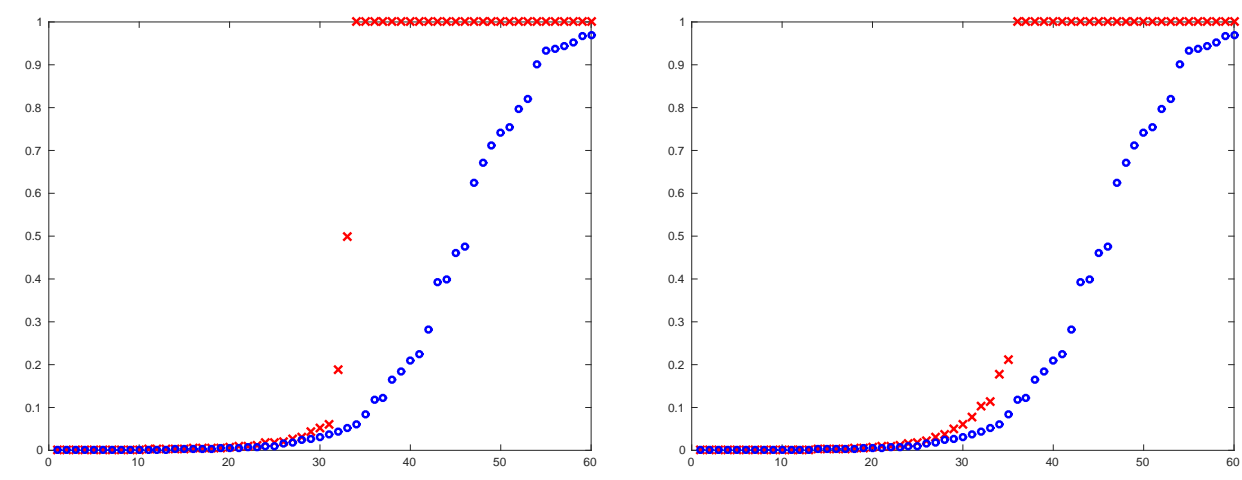

FIG. 5.4. A comparison of eigenvalues of $H^{-1}$ (blue circles) and $\tilde{H}^{-1}$ (red crosses) for two different eigenvalue combinations for MP1. Left: $N_{e}=(0,6,13,14), D=3.95 e-1$. Right: $N_{e}=(0,0,29,6), D=3.39 e-1$.

of the eigenvalues are close to one and have not been plotted). The corresponding eigenvalues of $\tilde{H}^{-1}$ for two different $N_{e}$ are plotted as red crosses. The values of $N_{e}$ used have been chosen to represent typical behavior: we observe that even when no information is retained at the finest grid levels, the overall shape of the eigenvalue distribution of $H^{-1}$ is still captured by the multilevel approximation.

As stated above, it is difficult to characterise a single 'best possible' eigenvalue combination. However, certain traits can be identified. Plots of the minimum distance achieved for various memory ratios $r$ are shown in Figure 5.5 for our four different test problems. In each subplot, the dashed line shows the minimum distance achieved for a given $r$, and the solid line shows the average over the $5 \%$ of eigenvalue combinations satisfying (2.14) which resulted in the smallest distances. The dotted line shows the equivalent distance achieved using only fine grid vectors. The key observation here is that when there is only room to store a small number of fine grid vectors in memory, using a multilevel approximation clearly gives much better accuracy. Although we did not identify an 'optimal' way of choosing $N_{e}$, our experiments did show that eigenvalue combinations which have no or very few eigenpairs on the finest level(s) appear to perform best. Based on this, we suggest the ansatz of doubling the number of eigenpairs calculated at each level (from fine to coarse grids). For example, the 

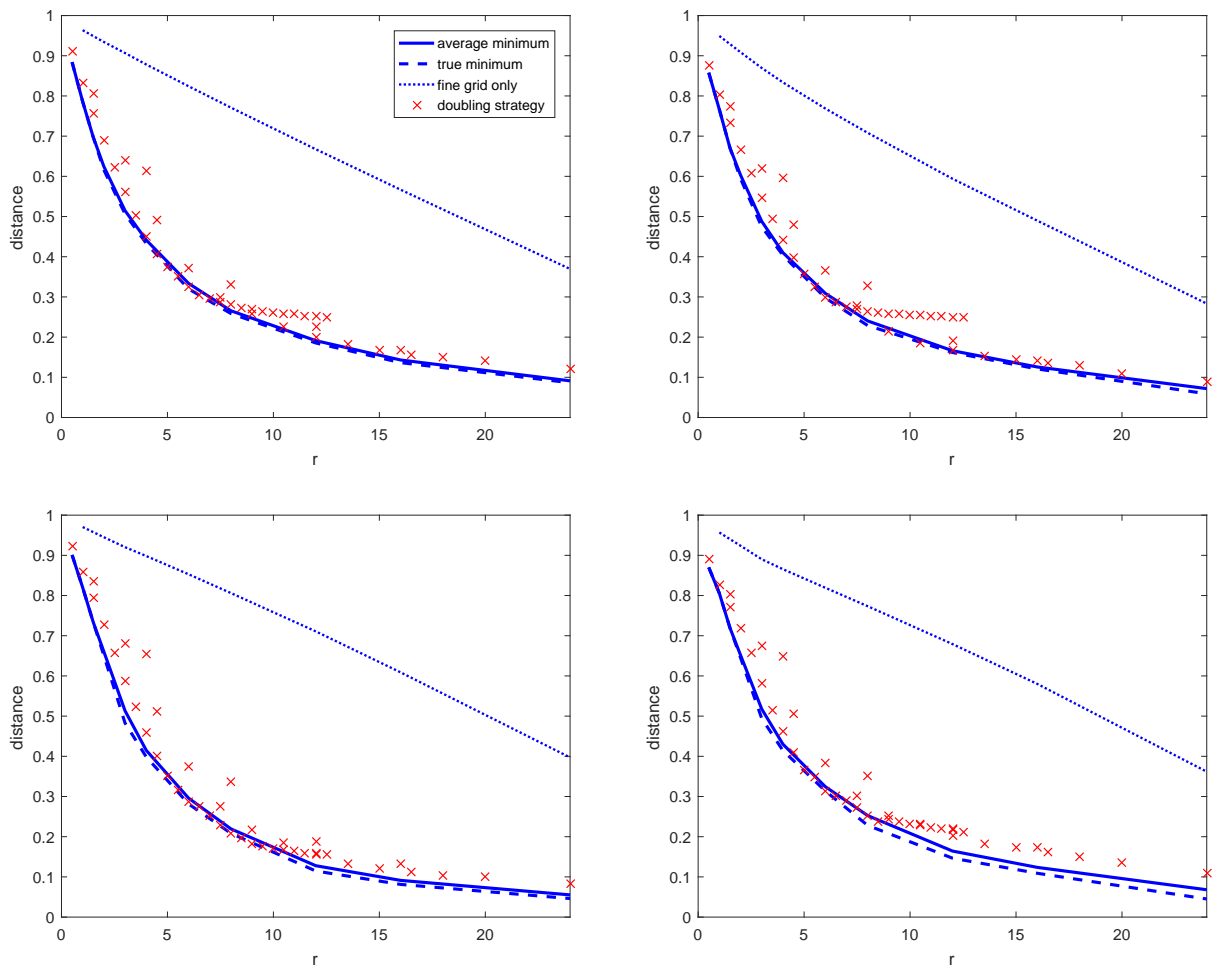

FIG. 5.5. Distance D (5.4) plotted against memory ratio $r$ for model problems MP1 (top left), MP2 (top right), MP3 (bottom left) and MP4 (bottom right).

combinations which correspond to $r=8$ are as follows: $N_{e}=(0,0,24,48)$ and $N_{e}=$ $(2,4,8,16)$. The distances achieved using this strategy for various values of $r$ are displayed in Figure 5.5 using red crosses. Although these combinations do not always give rise to the minimum distance, they usually give reasonable approximations. This doubling strategy will be adopted in the next section.

5.5. Using $\tilde{H}^{-1}$ as a preconditioner for Gauss-Newton. In the second set of experiments, we use our multilevel approximation to $H^{-1}$ in a typical application. Specifically, we recall the case mentioned in $\$ 1.2$ of incremental 4D-Var, where the solution of a system of linear equations of the form (1.9) with coefficient matrix $\mathcal{H}$ has to be approximated at each step of a Gauss-Newton process. This is typically achieved using a few CG iterations. In realistic DA applications, the number of Gauss-Newton (outer) iterations as well as the number of CG (inner) iterations is limited by the time available in the forecast window. More details of approximate Gauss-Newton methods for large-scale data assimilation problems can be found in [19].

After first-level preconditioning, system (1.9) takes the form

$$
H\left(u_{i}\right) \delta v_{i}=-\left(V_{b}^{1 / 2}\right)^{*} G\left(u_{i}\right),
$$

where $\delta v_{i}=V_{b}^{1 / 2} \delta u_{i}$. As we are assuming that this preconditioning is always applied, we will refer to this case as 'unpreconditioned Gauss-Newton. The convergence of the CG method applied to (5.5) can be further accelerated by preconditioning the system 


\begin{tabular}{cccccc} 
Precond. & Algorithm & $N_{e}$ & $k^{\prime}$ & $n_{k^{\prime}}^{l}$ & $N_{k^{\prime}}^{l}$ \\
\hline \hline P2a & 2 & $(200,0,0,0)$ & 1 & 8 & - \\
P2b & 2 & $(0,8,16,32)$ & 1 & 8 & - \\
P2c & 2 & $(0,4,8,16)$ & 1 & 8 & - \\
P3a & 3 & $(0,8,16,32)$ & 1 & 8 & $(0,0,8,0)$ \\
P3b & 3 & $(0,8,16,32)$ & 2 & 8 & $(0,0,0,8)$ \\
\hline \hline
\end{tabular}

TABLE 5.2

Description of parameters used in multilevel preconditioners.

again using $\tilde{H}^{-1}$ to improve the resulting eigenspectrum, in other words, by solving

$$
\tilde{H}^{-1}\left(u_{i}\right) H\left(u_{i}\right) \delta v_{i}=-\tilde{H}^{-1}\left(u_{i}\right)\left(V_{b}^{1 / 2}\right)^{*} G\left(u_{i}\right) .
$$

Here the preconditioner $\tilde{H}^{-1}\left(u_{i}\right)$ is computed once per Gauss-Newton step (before iterating with CG): this is an important difference from the approach presented in [11], where the multigrid cycle is applied as a preconditioner for each CG iteration. In what follows, we apply incremental 4D-Var to the Burgers' test problems, and investigate the effect of preconditioning using multilevel approximations $\tilde{H}^{-1}$ built using Algorithms 2 and 3. The Gauss-Newton stopping tolerance is $10^{-4}$, and 5 CG iterations are carried out for each linear solve. In each simulation, the multilevel preconditioner is not switched on until after the first few (five in our examples) Gauss-Newton iterations have been carried out. This is to ensure convergence of the outer iteration as our problem involves strongly nonlinear constraints, in the form of Burgers' equation (see, for example, [39]).

Details of the specific algorithms and eigenvalue combinations used for the preconditioners tested are given in Table 5.2. Preconditioners P2a, P2b and P2c use Algorithm 2 with different values of $N_{e}$ in the multilevel representation of the inverse Hessian $H^{-1}$ defined by (3.3). For each elementary Hessian $\hat{H}_{k^{\prime}}^{l}$, the Hessian-vector product is defined at level $k^{\prime}=1, m=201$, with $n_{k^{\prime}}^{l}=8$ eigenpairs used for its limited-memory representation. Preconditioners P3a and P3b use Algorithm 3 with one $N_{e}$ (such that $r=12$ ) in the multilevel representation of $H^{-1}$. For case P3a, the Hessian-vector product is again defined at level $k^{\prime}=1$, with $n_{k^{\prime}}^{l}=8$ eigenpairs used for limited-memory representation of $\left(\hat{H}_{k^{\prime}}^{l}\right)^{-1}$. The multilevel representation of $\hat{H}_{k^{\prime}}^{l}$ is then built according to $N_{k^{\prime}}^{l}$, that is, at level $k=2$. For case P3b, the Hessian-vector product is defined at level $k^{\prime}=2, m=101$, with $n_{k^{\prime}}^{l}=8$ eigenpairs used for limitedmemory representation of $\left(\hat{H}_{k^{\prime}}^{l}\right)^{-1}$. The multilevel representation of $\hat{H}_{k^{\prime}}^{l}$ is then built at level $k=3$ (the coarsest level). For each test case, the minimisation problem (1.2) was solved 25 times with perturbed data $u_{b}$ and $\varphi_{o b s}$ as described in [16, §5.2], and the resulting ensemble averaged values characterising the convergence rate and the solution accuracy are presented in convergence diagrams shown in Figures 5.6-5.9.

Each subplot of a diagram shows two major convergence indicators: the averaged deviation norm $\left\|u_{i}-u_{\infty}\right\|_{2}$ (where $u_{i}$ is the iterate after $i$ iterations and $u_{\infty}$ is the final estimate $\bar{u}$ ) and the gradient norm $\left\|G\left(u_{i}\right)\right\|_{2}$, plotted in $\log _{10}$-scale against computational time measured in two different units. These are the number of the cost-function/gradient evaluations and the number of Hessian-vector product evaluations (denoted by $N_{G}$ and $N_{H V P}$, respectively) at the finest discretisation level needed to carry out the whole solution procedure (that is, the cost of constructing and applying any preconditioning is included in these figures). The former coincides 

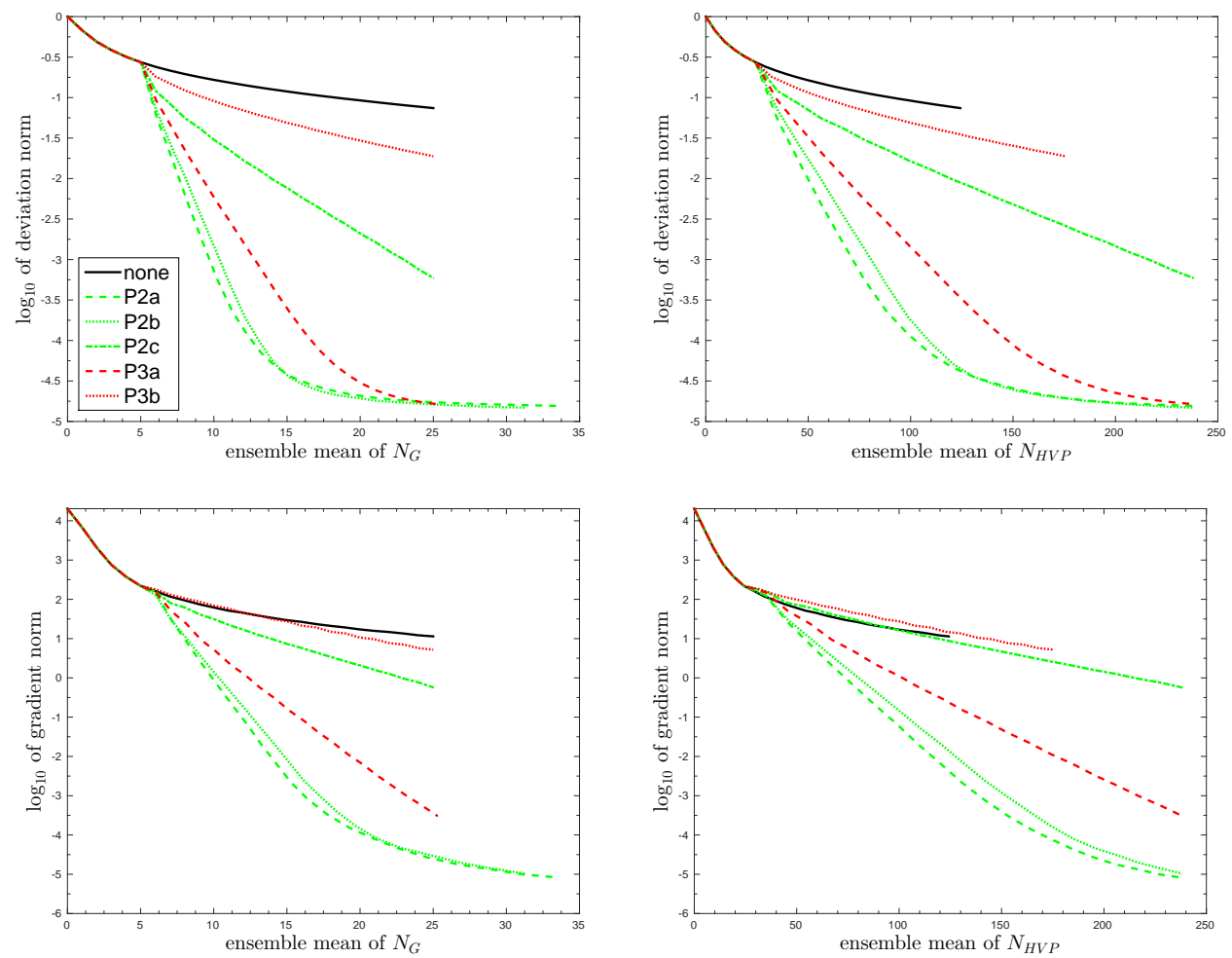

FIG. 5.6. Convergence diagram for MP1.

with the actual number of the cost-function/gradient evaluations, the latter also includes the maximum time spent on evaluating an individual $\hat{H}^{l}$ (we assume that $\hat{H}^{l}$ are evaluated in parallel).

To assess preconditioner performance, as compared to unpreconditioned GaussNewton, the convergence diagrams should be interpreted together as follows. Suppose that one cost-function/gradient evaluation takes time $t_{G}$, and one Hessian-vector product evaluation takes time $t_{H V P}$.

Let us denote $\epsilon=\log _{10}\left\|u_{i}-u_{\infty}\right\|_{2}$, then the relative accuracy of $u_{i}$ is $10^{\epsilon} \times 100 \%$. For a given relative accuracy the number of cost-function/gradient evaluations $N_{G}$ required to achieve it with a given preconditioner can be read from the top left subplot as the abscissa of the point where the level line $\epsilon$ crosses the relevant curve. The value of the gradient norm $\varepsilon=\log _{10}\left\|G\left(u_{i}\right)\right\|_{2}$ which corresponds to this $\epsilon$ can then be read from the lower left subplot as the value at this abscissa. The number of required Hessian-vector products $N_{H V P}$ is available from the top right subplot in a similar way. The resulting values $N_{G}$ and $N_{H V P}$ can then be used to evaluate the total time required as $t_{\text {total }}=N_{G} \times t_{G}+N_{H V P} \times t_{H V P}$. Since one unpreconditioned GaussNewton iteration takes a time of $t=t_{G}+N_{C G} \times t_{H V P}$, this total amount of time can be spent on $i^{*}$ unpreconditioned iterations where

$$
i^{*}(\rho)=\frac{t_{\text {total }}}{t}=\frac{\rho N_{G}+N_{H V P}}{\rho+N_{C G}}
$$

and $\rho=t_{G} / t_{H V P}$. Once $i^{*}$ has been identified, the accuracy $\epsilon^{*}$ achieved by unpreconditioned Gauss-Newton in the same time as the preconditioned version gives an 

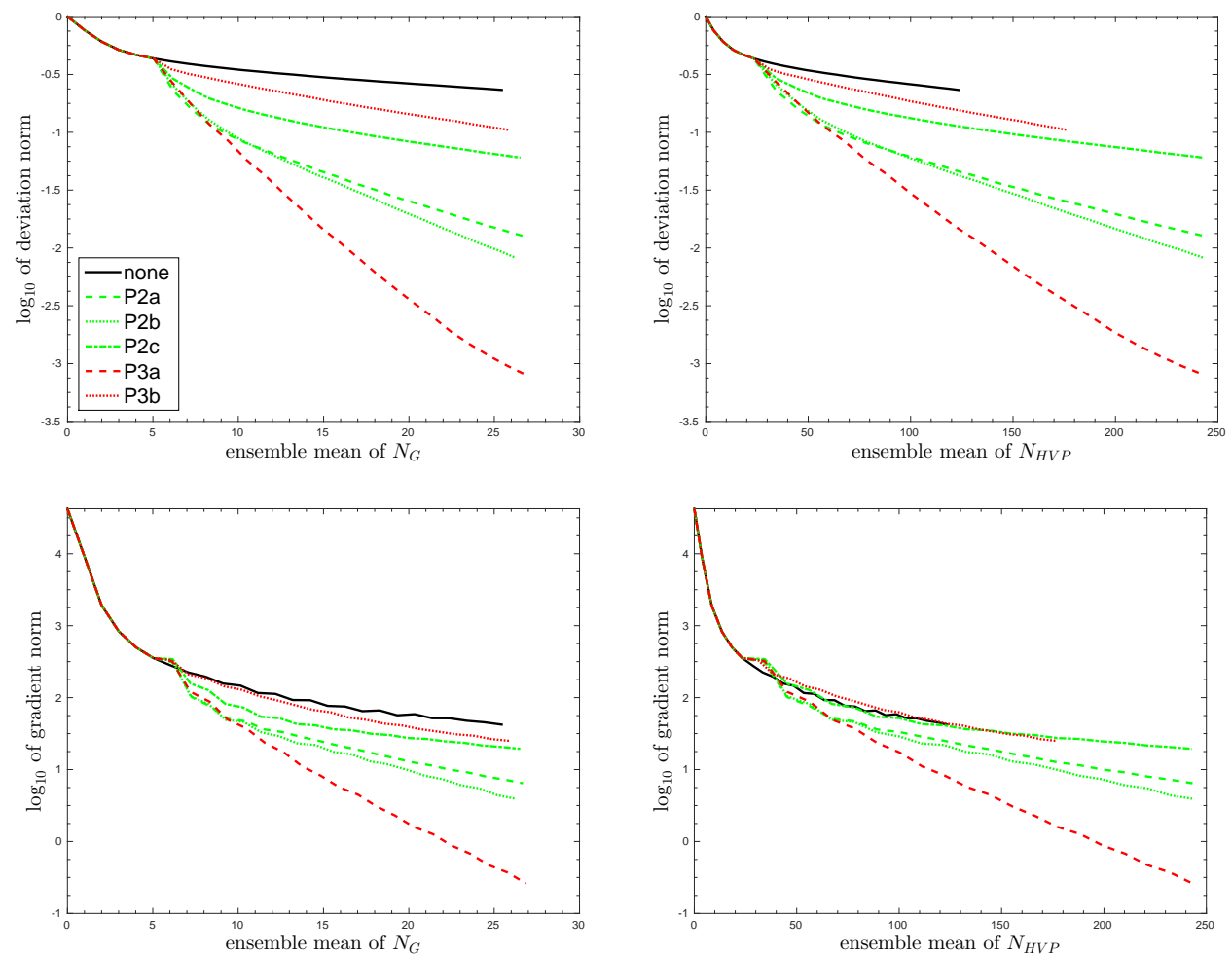

FIG. 5.7. Convergence diagram for MP2.

accuracy of $\epsilon$, can be read off from the top left subplot as the value from the curve labelled 'none at the point $N_{G}=i^{*}$. The difference $\epsilon-\epsilon^{*}$ reflects the accuracy gain due to preconditioning, achieved in the time $i^{*} \times t_{G}$. The value of the gradient norm $\varepsilon^{*}$ achieved by unpreconditioned Gauss-Newton in the same time as the preconditioned version gives the gradient norm $\varepsilon$ can also be read from the bottom left subplot as the value of the corresponding graph at $N_{G}=i^{*}$. The difference $\varepsilon-\varepsilon^{*}$ provides the convergence gain due to preconditioning, achieved in the time $i^{*} \times t_{G}$. Alternatively, the same procedure can be replicated starting from a desired gradient norm reduction defined by $\varepsilon$, in which case the bottom right subplot will be involved.

Some results of such analysis with $\rho=1$ and $\epsilon=-2$ are summarised in Table 5.3, which shows the relative accuracy achieved by unpreconditioned GN by time preconditioned GN achieved relative accuracy $1 \%$. For case MP2, when the values cannot be identified from the figures shown, the table fields are empty, and where the values have been obtained by extrapolation, they are marked as approximate. The larger the value in Table 5.3, the less accuracy has been achieved by using unpreconditioned Gauss-Newton method, and the more efficient the chosen multilevel preconditioner has been. Note that $\rho \approx 1$ relates to the case where computational time for solving the original non-linear model and the tangent linear model is comparable. Similar tables can be obtained for different values of $\rho$ and $\epsilon$.

These results demonstrate that, given a fixed computational time, the optimal solution accuracy gain due to multilevel preconditioning is about an order of magnitude. Therefore, the suggested approach may be considered as an important resource for parallelisation of the minimisation process itself. It is clear that for the variants 

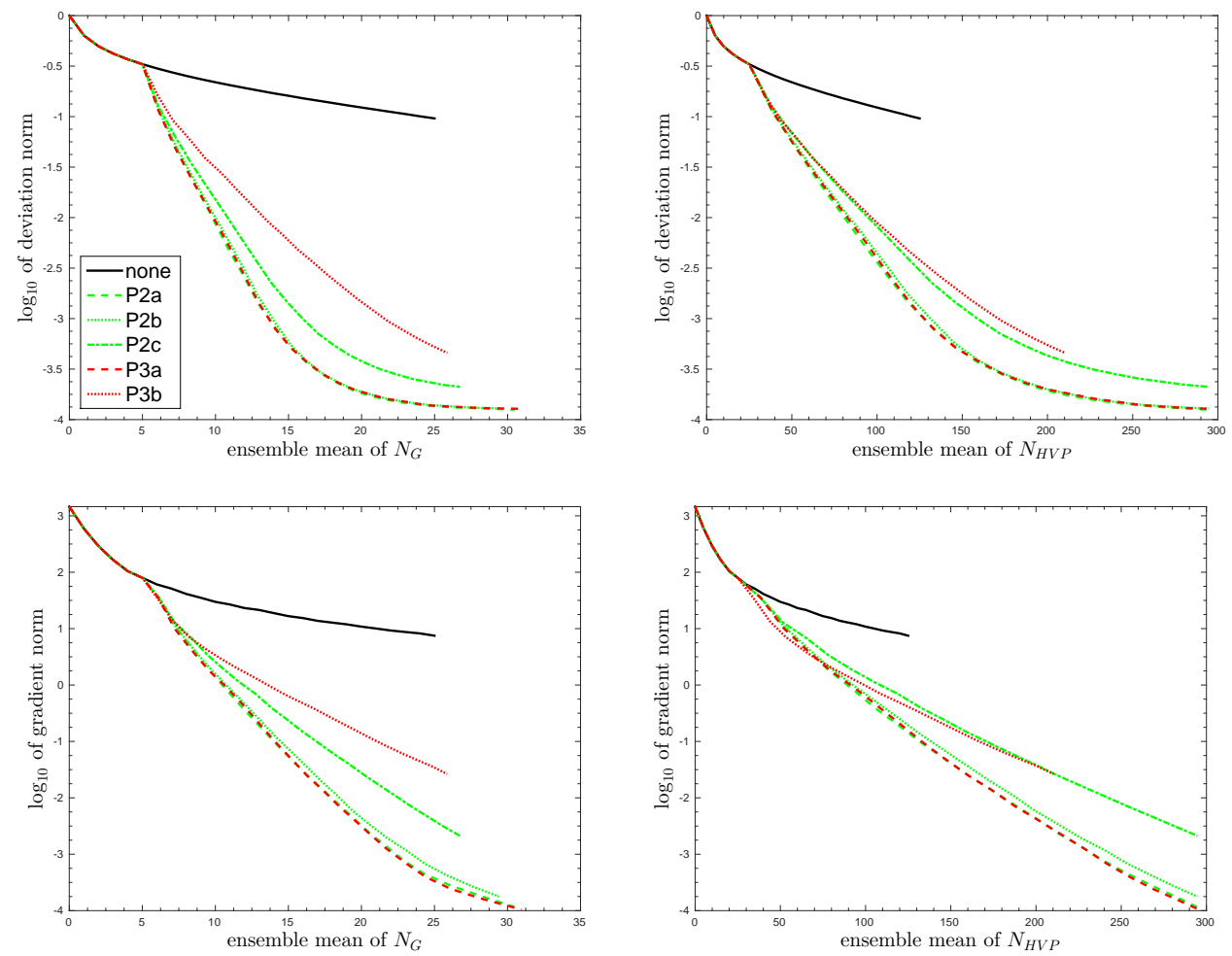

FIG. 5.8. Convergence diagram for MP3.

\begin{tabular}{ccccc} 
Precond. & case MP1 & case MP2 & case MP3 & case MP4 \\
\hline \hline P2a & $15.8 \%$ & $15.7 \%$ & $12.5 \%$ & $12.0 \%$ \\
P2b & $13.1 \%$ & $17.5 \%$ & $12.5 \%$ & $12.0 \%$ \\
P2c & $6.3 \%$ & - & $10.0 \%$ & $10.0 \%$ \\
P3a & $10.0 \%$ & $20.0 \%$ & $12.5 \%$ & $12.0 \%$ \\
P3b & $\approx 3.0 \%$ & - & $9.0 \%$ & $\approx 7.0 \%$ \\
\hline \hline
\end{tabular}

TABLE 5.3

Relative accuracy achieved by unpreconditioned GN by time preconditioned GN achieved relative accuracy $1 \%$.

using Algorithm 2, the performance with $\mathrm{P} 2 \mathrm{a}$ and $\mathrm{P} 2 \mathrm{~b}$ is quite similar, that is, $\mathrm{P} 2 \mathrm{~b}$ essentially replicates the performance of the full eigenvalue decomposition based P2a, with much-reduced memory requirements $(r=12$ as opposed to $r=200)$. With MP1, the performance of preconditioner P2c $(r=6)$ degrades, as not enough information has been retained at the local level (although it still represents a significant improvement over the unpreconditioned version). However, in case MP3 preconditioner P2c performs nearly as well as P2b. As discussed in $\S 5.3$, there is a difference between stationary and moving sensors in terms of their influence on the inverse Hessian structure in that, in the case of moving sensors, a much better quality multilevel representation can be achieved within the same memory allowance. The performance of P2c in cases MP3 and MP4 certainly confirms this. For Algorithm 3, as expected, the additional level of approximation in P3a means that it does not perform quite as well as P2a in 

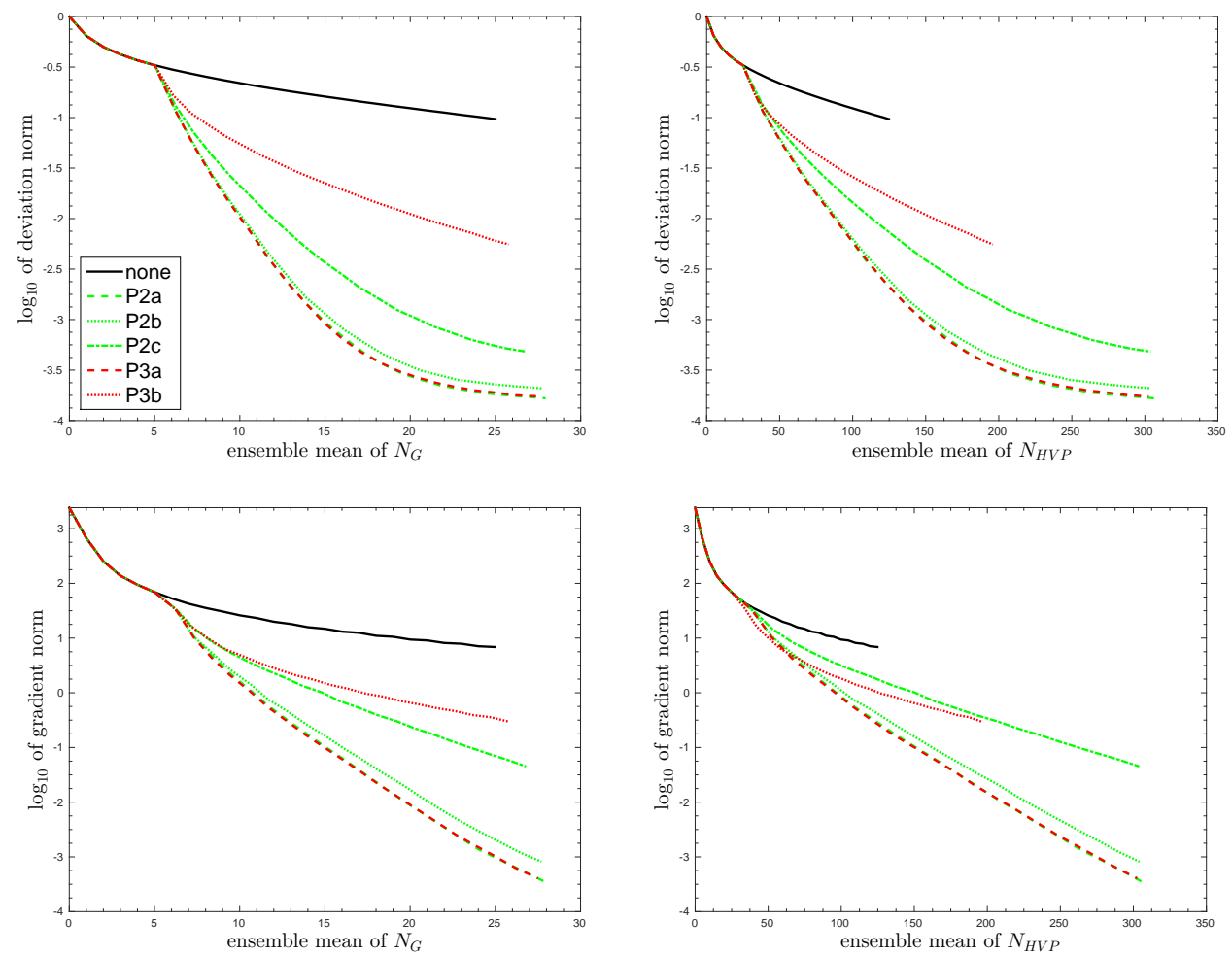

FIG. 5.9. Convergence diagram for MP4.

cases MP1 and MP2, but it is still a very competitive method. With preconditioner P3b in cases MP1 and MP2, however, it appears that the local Hessian calculations have been done on too coarse a grid so too much information has been lost. At the same time, P3b performs very well in cases MP3 and MP4 (with convergence curves almost indistinguishable from those of $\mathrm{P} 2 \mathrm{a}$ ), which is another indication that this type of preconditioning could be very useful in assimilating satellite data.

It is interesting to note that in case MP2, method P3a performs better than $\mathrm{P} 2 \mathrm{a}$, which on the surface seems out of line with the other results. However, from Figure 5.1(right) it can be seen that the flow solution for initial state $u_{\text {true }}^{2}$ contains much stronger field gradients than in case $u_{\text {true }}^{1}$. This is combined with singular-type data from stationary sensors in case MP1, which explains slower convergence of the Gauss-Newton process in comparison to all other cases. For linear model equations, the Gauss-Newton method must converge in one iteration if the inner problem (1.9) is solved exactly. In the case of non-linear model equations, many Gauss-Newton iterations are needed, and solving the inner problem too accurately has rather a negative impact on the Gauss-Newton convergence rate. This explains the better performance with a less accurate approximation as preconditioner, as seen with P3a in case MP2.

6. Conclusion. The inverse Hessian of the auxiliary DA problem (1.5) plays an important part in different aspects of variational DA. The Hessian-vector product is defined by sequential solution of the tangent linear and adjoint problems; for the inverse Hessian (or its square-root), no such definition is possible. In high dimensions, 
the requirement to work in a matrix-free environment means that compact representation schemes are of significant interest. The simplest one, based on the eigenvalue decomposition of the projected Hessian after first-level preconditioning, is however not good enough. Here we have introduced the novel concept of a multilevel eigenvalue decomposition, which results in a much more efficient compact representation of the inverse Hessian (and its square root). At a given level, the eigensystem of an operator preconditioned by its own approximation from the next coarser level is computed, ascending from the coarsest to the finest level. The numerical results in $§ 5.4$ demonstrated that, given a specified memory allowance, the inverse Hessian approximation accuracy is greatly improved as compared to a one-level eigenvalue decomposition scheme. We also believe that a similar algorithm can be utilised beyond the application considered here, for example, for any symmetric operator resulting from the discretisation of a PDE, or for image compression and restoration. In such cases, the resulting low-memory representations of the inverse operator could be particularly useful for preconditioning in problems with multiple right-hand sides.

We have also considered the application of our compact inverse Hessian approximations as preconditioners for a Gauss-Newton minimisation procedure. Here we introduced a further novel decomposition principle, namely, allowing the Hessian to be represented by the sum of elementary Hessians, which can be evaluated (and compressed) in parallel. The numerical results in Section 5.5 show that, given a fixed execution time, a much more accurate approximation can be computed as compared to using unpreconditioned Gauss-Newton method. The new multilevel method therefore offers an important parallelisable resource applicable directly to minimisation problems. This offers a significant advantage over the multigrid approach in [11], which is intrinsically sequential.

The applicability of our method in the context of present day operational 4D-Var systems depends, in theory, on the eigenvalue structure of the associated Hessians (local and global). In practice, it will also depend on many different factors which are difficult to assess at this stage. As an intermediate step, we intend to test our method with a global shallow water equations (SWE) model defined on a sphere [33]. This will allow us to consider a few features which are not involved with the current Burgers' model. In particular, the SWE model is a full two-dimensional model which includes 3 state variables and also supports long wave transport (rather than advection/diffusion). We are hopeful that our new approach will also work well in this more realistic situation.

Appendix A. The tangent linear model $R^{\prime}(u) v=\psi$ for Burgers' equation (5.1) is given by

$$
\left\{\begin{array}{l}
\frac{\partial \psi}{\partial t}=-\frac{\partial(\varphi \psi)}{\partial x}+\frac{\partial}{\partial x}\left(\mu(\varphi) \frac{\partial \psi}{\partial x}\right)+\frac{\partial}{\partial x}\left(\mu^{\prime}(\varphi) \psi \frac{\partial \varphi}{\partial x}\right) \\
\left.\frac{\partial \psi}{\partial x}\right|_{x=0,1}=0 \\
\left.\psi\right|_{t=0}=v
\end{array}\right.
$$

where $\varphi=\varphi(u, x, t), \psi=\psi(x, t), x \in[0,1], t \in[0, T]$. The associated adjoint model 
$R^{\prime *}(u) \psi=v$ is

$$
\left\{\begin{array}{l}
-\frac{\partial \psi^{*}}{\partial t}=\varphi \frac{\partial \psi^{*}}{\partial x}+\frac{\partial}{\partial x}\left(\mu(\varphi) \frac{\partial \psi^{*}}{\partial x}\right)-\mu^{\prime}(\varphi) \frac{\partial \varphi}{\partial x} \frac{\partial \psi^{*}}{\partial x}+\psi \\
\left.\left(\mu(\varphi) \frac{\partial \psi^{*}}{\partial x}+\varphi \psi^{*}\right)\right|_{x=0,1}=0 \\
\left.\psi^{*}\right|_{t=T}=0 \\
\left.\psi^{*}\right|_{t=0}=v
\end{array}\right.
$$

where $\varphi=\varphi(u, x, t), \psi^{*}=\psi^{*}(x, t), \psi=\psi(x, t), x \in[0,1], t \in[T, 0]$.

Acknowledgements. The corresponding author acknowledges initial funding for this project through the UK Natural Environment Research Council (NERC grant NE/J018201/1) and also thanks Prof. Achi Brandt from the Weizmann Institute of Science, Israel for joint work on using multigrid methods for solving control problems.

\section{REFERENCES}

[1] T. Amemiya, Non-linear regression models. in Handbook of Econometrics, North-Holland Publishing Company, Amsterdam, 2002.

[2] W. ARnoldi, The principle of minimized iterations in the solution of the matrix eigenvalue problem, Quarterly of Applied Mathematics, 9 (1951), pp. 17-29.

[3] N. BAKHVAlov, On the convergence of a relaxation method with natural constraints on the elliptic operator, USSR Comp. Math. Math. Phys., 6 (1966), pp. 101-113.

[4] A. Borzi And V. Schulz, Multigrid methods for pde optimization, SIAM Review, 51 (2009), pp. 361-395.

[5] A. Brandt, Multi-level adaptive solutions to boundary value problems, Math. Comp., 31 (1977), pp. 333-390.

[6] Y. Chen And D. Oliver, Ensemble randomized maximum likelihood method as an iterative ensemble smoother, Math. Geosci., 44 (2012), pp. 1-26.

[7] S. Costiner And S. TA'ASAn, Adaptive multigrid techniques for large-scale eigenvalue problems: solutions of the schrodinger problem in two and three dimensions, Phys. Rev., 44 (1995), pp. 3704-3717.

[8] P. Courtier, J. Thepaut, and A. Hollingsworth, A strategy for operational implementation of 4d-var, using an incremental approach, Quart. J. Roy. Meteor. Soc., 120 (1994), pp. $1367-1387$.

[9] J. Cullum And R. Willoughby, Lanczos Algorithms for Large Symmetric Eigenvalue Computations: Vol. I: Theory, Classics in Applied Mathematics, SIAM, Philadelphia, 2002.

[10] M. Dashti, K. Law, A. Stuart, And J. Voss, Map estimators and posterior consistency in bayesian nonparametric inverse problems, Inverse Problems, 29 (2013), p. 095017.

[11] L. Debreu, E. Neveu, E. Simon, F.-X. L. Dimet, and A. Vidard, Multigrid solvers and multigrid preconditioners for the solution of variational data assimilation problems, Quart. J. Roy. Meteor. Soc., 142 (2016), pp. 515-528.

[12] F.-X. L. Dimet and O. Talagrand, Variational algorithms for analysis and assimilation of meteorological observations: theoretical aspects, Tellus A, 38 (1986), pp. 97-110.

[13] M. Ehrendorfer and J. Tribbia, Optimal prediction of forecast error covariances through singular vectors, J. Atmos. Sci., 54 (1997), pp. 286-313.

[14] E. Epstein, The role of initial uncertainties in prediction, J. Appl. Meteor., 8 (1969), pp. 190198.

[15] D. Furbish, M. Hussaini, F.-X. L. Dimet, P. Ngnepieba, and Y. Wu, On discretization error and its control in variational data assimilation, TELLUS A, 60 (2008), pp. 979-991.

[16] I. Gejadze, F.-X. L. Dimet, and V. Shutyaev, On analysis error covariances in variational data assimilation, SIAM J. Sci. Comput., 30 (2008), pp. 1847-1874.

[17] - On optimal solution error covariances in variational data assimilation problems, J. Comput. Phys., 229 (2010), pp. 2159-2178.

[18] - Computation of the analysis error covariance in variational data assimilation problems with nonlinear dynamics, J. Comput. Phys., 230 (2011), pp. 7923-7943. 
[19] S. Gratton, A. Lawless, and N. Nichols, Approximate Gauss-Newton methods for nonlinear least squares problems, SIAM J. Optim., 18 (2007), pp. 106-132.

[20] S. Haben, A. Lawless, And N. Nichols, Conditioning and preconditioning of the variational data assimilation problem, Computers \& Fluids, 46 (2011), pp. 252-256.

[21] W. HackBusch, On the computation of approximate eigenvalues and eigenfunctions of elliptic operators by means of a multigrid method, SIAM J. Numer. Anal., 16 (1979), pp. 201-215.

[22] L. Hascoet and V. Pascual, Tapenade 2.1 User's Guide, INRIA Technical Report, Sophia Antipolis, 2004.

[23] M. Hestenes and E. Stiefel, Methods of conjugate gradients for solving linear systems, J. Res. Natl Bur. Stand., 49 (1952), pp. 409-436.

[24] T. HWAng And I. Parsons, A multigrid method for the generalized symmetric eigenvalue problem: part $i$ - algorithm and implementation, Int. J. Numer. Meth. Eng., 35 (1992), pp. 1663-1676.

[25] A. Knyazev And K. Neymeyr, Efficient solution of symmetric eigenvalue problems using multigrid preconditioners in the locally optimal block conjugate gradient method, ETNA: Electronic transactions on Numerical Analysis, 15 (2003), pp. 38-55.

[26] C. LANCzos, An iteration method for the solution of the eigenvalue problem of linear differential and integral operators, J. Res. Natl Bur. Std., 45 (1950), pp. 225-282.

[27] R. B. LehoucQ, D. C. Sorensen, And C. YAng, ARPACK users guide: Solution of large scale eigenvalue problems by implicitly restarted Arnoldi methods., 1997.

[28] J.-L. Lions, Contrôle Optimal des Systèmes Gouvernés par des Équations aux Dérivées Partielles, Dunod, Paris, 1968.

[29] C. Liu, Q. XiaO, AND B. WANG, An ensemble-based four-dimensional variational data assimilation scheme. part $i$ : Technical formulation and preliminary test., Mon. Weather Rev., 136 (2008), pp. 3363-3373.

[30] G. Marchuk, V. Agoshrov, and V. Shutyaev, Adjoint Equations and Perturbation Algorithms in Nonlinear Problems, CRC Press Inc., New-York, 1996.

[31] I. Mirouze And A. Weaver, Representation of correlation functions in variational assimilation using an implicit diffusion operator, Q.J.R. Meteorol. Soc., 136 (2010), pp. 1421-1443.

[32] M. MoAkHer, A differential geometric approach to the geometric mean of symmetric positivedefinite matrices, SIAM J. Matrix Anal. Appl., 26 (2005), pp. 735-747.

[33] I. NAVON AND J. YU, Exshall: A turkel-zwas explicit large time-step fortran programm for solving the shallow water equations in spherical co-ordinates, Computers and Geosciences, 17 (1991), pp. 1311-1343.

[34] S. PatankaR, Numerical Heat Transfer and Fluid Flow, Hemisphere Publishing Corporation, New-York, 1980.

[35] F. Rabier AND P. Courtier, Four-dimensional assimilation in the presence of baroclinic instability, Quart. J. Roy. Meteorol. Soc., 118 (1992), pp. 649-672.

[36] F. Rabier, H. Järvinen, E. Klinker, J.-F. Mahfouf, and A. Simmons, The ecmwf operational implementation of four-dimensional variational assimilation. $i$ : Experimental results with simplified physics, Quart. J. Roy. Meteorol. Soc., 126 (1992), pp. 1142-1170.

[37] G. Sleijpen And H. V. DER Vorst, A jacobi-davidson iteration method for linear eigenvalue problems, SIAM J. Matrix Anal. Appl., 17 (1996), pp. 401-425.

[38] W. ThaCKer, The role of the Hessian matrix in fitting models to measurements, J. Geophys. Res., 94 (1989), pp. 6177-6196.

[39] R. A. to Solving Large Residual Nonlinear Least Squares Problems, Siam Review, 22 (1980), pp. 1-11.

[40] Z. Toth And E. Kalnay, Ensemble forecasting at NMC: The generation of perturbations, Bull. Amer. Meteor. Soc., (1993), pp. 2317-2330.

[41] U. Trottenberg, C. Oosterlee, and A. Schller, Multigrid, Academic Press, London, 2001. 